\title{
On the class of caustics by reflection of planar curves
}

\author{
Alfrederic Josse And FrançoIse PÈne
}

\begin{abstract}
Given any light position $S \in \mathbb{P}^{2}$ and any algebraic curve $\mathcal{C}$ of $\mathbb{P}^{2}$ (with any kind of singularities), we consider the incident lines coming from $S$ (i.e. the lines containing $S$ ) and their reflected lines after reflection on the mirror curve $\mathcal{C}$. The caustic by reflection $\Sigma_{S}(\mathcal{C})$ is the Zariski closure of the envelope of these reflected lines. We introduce the notion of reflected polar curve and express the class of $\Sigma_{S}(\mathcal{C})$ in terms of intersection numbers of $\mathcal{C}$ with the reflected polar curve, thanks to a fundamental lemma established in [16]. This approach enables us to state an explicit formula for the class of $\Sigma_{S}(\mathcal{C})$ in every case in terms of intersection numbers of the initial curve $\mathcal{C}$.
\end{abstract}

Mathematics Subject Classification (2010): 14H50 (primary); 14E05, 14N05, $14 \mathrm{~N} 10$ (secondary).

\section{Introduction}

Let $\mathbf{V}$ be a three dimensional complex vector space endowed with some fixed basis. We consider a light point $S\left[x_{0}: y_{0}: z_{0}\right] \in \mathbb{P}^{2}:=\mathbb{P}(\mathbf{V})$ and a mirror given by an irreducible algebraic curve $\mathcal{C}=V(F)$ of $\mathbb{P}^{2}$, with $F \in \operatorname{Sym}^{d}\left(\mathbf{V}^{\vee}\right)(F$ corresponds to a polynomial of degree $d$ in $\mathbb{C}[x, y, z])$. We denote by $d^{\vee}$ the class of $\mathcal{C}$. We consider the caustic by reflection $\Sigma_{S}(\mathcal{C})$ of the mirror curve $\mathcal{C}$ with source point $S$. Recall that $\Sigma_{S}(\mathcal{C})$ is the Zariski closure of the envelope of the reflected lines associated to the incident lines coming from $S$ after reflection off $\mathcal{C}$. When $S$ is not at infinity, Quetelet and Dandelin $[9,18]$ proved that the caustic by reflection $\Sigma_{S}(\mathcal{C})$ is the evolute of the $S$-centered homothety (with ratio 2 ) of the pedal of $\mathcal{C}$ from $S$ (i.e. the evolute of the orthotomic of $\mathcal{C}$ with respect to $S$ ). This decomposition has also been used in a modern approach by [2-4] to study the source genericity (in the real case). In [16] we proved formulas for the degree of the caustic by reflection of planar algebraic curves. For a presentation of the classical notions of envelope, evolute, pedal, contrapedal, orthotomic, we refer to $[4,8,10,20,23]$.

In [7], Chasles proved that the class of $\Sigma_{S}(\mathcal{C})$ is equal to $2 d^{\vee}+d$ for a generic $(\mathcal{C}, S)$. In [1], Brocard and Lemoyne gave (without any proof) a more general 
formula only when $S$ is not at infinity. The Brocard and Lemoyne formula appears to be the direct composition of formulas got by Salmon and Cayley in [19, page 137, 154] for some geometric characteristics of evolute and pedal curves. The formula given by Brocard and Lemoyne is not satisfactory for the following reasons. The results of Salmon and Cayley apply only to curves having no singularities other than ordinary nodes and cusps [19, page 92], but the pedal of such a curve is not necessarily a curve satisfying the same properties. For example, the pedal curve of the rational cubic $V\left(y^{2} z-x^{3}\right)$ from $[4: 0: 1]$ is a quartic curve with an ordinary triple point. Therefore it is not correct to compose directly the formulas got by Salmon and Cayley as Brocard and Lemoyne apparently did (see also Section 5 for a counterexample of the Brocard and Lemoyne formula for the class of the caustic by reflection).

Let us mention some works on the evolute and on its generalization in higher dimension $[6,11,21]$. In [11], Fantechi gave a necessary and sufficient condition for the birationality of the evolute of a curve and studied the number and type of the singularities of the general evolute. Let us insist on the fact that there exist irreducible algebraic curves (other than lines and circles) for which the evolute map is not birational. This study of evolute is generalized in higher dimension by Trifogli in [21] and by Catanese and Trifogli [6].

The aim of the present paper is to give a formula for the class (with multiplicity) of the caustic by reflection for any algebraic curve $\mathcal{C}$ (without any restriction neither on the singularity points nor on the flex points) and for any light position $S$ (including the case when $S$ is at infinity or when $S$ is on the curve $\mathcal{C}$ ).

In Section 1, we define the reflected lines $\mathcal{R}_{m}$ at a generic $m \in \mathcal{C}$ and the (rational) "reflected map" $R_{\mathcal{C}, S}: \mathbb{P}^{2} \rightarrow \mathbb{P}^{2}$ mapping a generic $m \in \mathcal{C}$ to the equation of $\mathcal{R}_{m}$.

In Section 2, we define the caustic by reflection $\Sigma_{S}(\mathcal{C})$, we give conditions ensuring that $\Sigma_{S}(\mathcal{C})$ is an irreducible curve and we prove that its class is the degree of the image of $\mathcal{C}$ by $R_{\mathcal{C}, S}$.

In Section 3, we give formulas for the class of caustics by reflection valid for any $(\mathcal{C}, S)$. These formulas describe precisely how the class of the caustic depends on geometric invariants of $\mathcal{C}$ and also on the relative positions of $S$ and of the two cyclic points $I, J$ with respect to $\mathcal{C}$. As a consequence of this result, we obtain the following formula for the class of $\Sigma_{S}(\mathcal{C})$ valid for any $\mathcal{C}$ of degree $d \geq 2$ and for a generic source position $S$ :

$$
\operatorname{class}\left(\Sigma_{S}(\mathcal{C})\right)=2 d^{\vee}+d-\Omega\left(\mathcal{C}, \ell_{\infty}\right)-\mu_{I}(\mathcal{C})-\mu_{J}(\mathcal{C}),
$$

where $\Omega\left(\mathcal{C}, \ell_{\infty}\right)$ is the contact number of $\mathcal{C}$ with the line at infinity $\ell_{\infty}$ and where $\mu_{I}(\mathcal{C})$ and $\mu_{J}(\mathcal{C})$ denote the multiplicity numbers of respectively $I$ and $J$ on $\mathcal{C}$.

In Section 4, our formulas are illustrated on two examples of curves (the lemniscate of Bernoulli and the quintic considered in [16]).

In Section 5, we compare our formula with the one given by Brocard and Lemoyne for a light position not at infinity. We also give an explicit counterexample to their formula. 
In Section 6, we prove our main theorem. First we give a formula for the class of the caustic in terms of intersection numbers of $\mathcal{C}$ with a generic "reflected polar" at the base points of $R_{\mathcal{C}, S}$. We then compute these intersection numbers in terms of the degree $d$ and of the class $d^{\vee}$ of $\mathcal{C}$ but also in terms of intersection numbers of $\mathcal{C}$ with each line of the triangle $I J S$.

In appendix $\mathrm{A}$, we prove a useful formula expressing the classical intersection number in terms of probranches.

ACKNOWLEDGEMENTS. The authors thank Jean Marot for stimulating discussions and for having indicated them the formula of Brocard and Lemoyne.

\section{Reflected lines $\mathcal{R}_{m}$ and rational map $R_{\mathcal{C}, S}$}

Recall that we consider a light position $S\left[x_{0}: y_{0}: z_{0}\right] \in \mathbb{P}^{2}$ and an irreducible algebraic (mirror) curve $\mathcal{C}=V(F)$ of $\mathbb{P}^{2}$ given by a homogeneous polynomial $F \in \operatorname{Sym}^{d}(\mathbf{V})$ with $d \geq 2$. We write $\operatorname{Sing}(\mathcal{C})$ for the set of singular points of $\mathcal{C}$. For any non singular point $m$, we write $\mathcal{T}_{m} \mathcal{C}$ for the tangent line to $\mathcal{C}$ at $m$. We set $\mathbf{S}\left(x_{0}, y_{0}, z_{0}\right) \in \mathbf{V} \backslash\{\mathbf{0}\}$. For any $m[x: y: z] \in \mathbb{P}^{2}$, we write $\mathbf{m}(x, y, z) \in \mathbf{V} \backslash\{\mathbf{0}\}$. We write as usual $\ell_{\infty}=V(z) \subset \mathbb{P}^{2}$ for the line at infinity. For any $\mathbf{P}\left(x_{1}, y_{1}, z_{1}\right) \in$ $\mathbf{V} \backslash\{\mathbf{0}\}$, we define

$$
\Delta_{\mathbf{P}} F:=x_{1} F_{x}+y_{1} F_{y}+z_{1} F_{z} \in \operatorname{Sym}^{d-1}\left(\mathbf{V}^{\vee}\right) .
$$

Recall that $V\left(\Delta_{\mathbf{P}} F\right)$ is the polar curve of $\mathcal{C}$ with respect to $P\left[x_{1}: y_{1}: z_{1}\right] \in \mathbb{P}^{2}$.

Since the initial problem is euclidean, we endow $\mathbb{P}^{2}$ with an angular structure for which $I[1: i: 0] \in \mathbb{P}^{2}$ and $J[1:-i: 0] \in \mathbb{P}^{2}$ play a particular role. To this end, let us recall the definition of the cross-ratio $\beta$ of 4 points of $\ell_{\infty}$. Given four points $\left(P_{i}\left[a_{i}: b_{i}: 0\right]\right)_{i=1, \ldots, 4}$ such that each point appears at most 2 times, we define the cross-ratio $\beta\left(P_{1}, P_{2}, P_{3}, P_{4}\right)$ of these four points as follows:

$$
\beta\left(P_{1}, P_{2}, P_{3}, P_{4}\right)=\frac{\left(b_{3} a_{1}-b_{1} a_{3}\right)\left(b_{4} a_{2}-b_{2} a_{4}\right)}{\left(b_{3} a_{2}-b_{2} a_{3}\right)\left(b_{4} a_{1}-b_{1} a_{4}\right)},
$$

with convention $\frac{1}{0}=\infty$. For any distinct lines $\mathcal{A}_{1}$ and $\mathcal{A}_{2}$ not equal to $\ell_{\infty}$, containing neither $I$ nor $J$, we define the oriented angular measure between $\mathcal{A}_{1}$ and $\mathcal{A}_{2}$ by $\theta$ (modulo $\pi \mathbb{Z}$ ) such that

$$
e^{-2 i \theta}=\beta\left(P_{1}, P_{2}, I, J\right)=\frac{\left(a_{1}+i b_{1}\right)\left(a_{2}-i b_{2}\right)}{\left(a_{1}-i b_{1}\right)\left(a_{2}+i b_{2}\right)}
$$

(where $P_{i}\left[a_{i}: b_{i}: 0\right]$ is the point at infinity of $\left.\mathcal{A}_{i}\right)$. Let $Q \in \operatorname{Sym}^{2}\left(\mathbf{V}^{\vee}\right)$ be defined by $Q(x, y, z):=x^{2}+y^{2}$. It will be worth noting that $Q(\nabla F)=F_{x}^{2}+$ 
$F_{y}^{2}=\Delta_{I} F \Delta_{J} F$. For every non singular point $m$ of $\mathcal{C} \backslash \ell_{\infty}$, we recall that $t_{m}\left[F_{y}\right.$ : $\left.-F_{x}: 0\right] \in \mathbb{P}^{2}$ is the point at infinity of $\mathcal{T}_{m} \mathcal{C}$ and so $t_{m} \notin\{I, J\}$ is equivalent to $m \notin V(Q(\nabla F))$.

Now, for any $m \in \mathcal{C} \backslash\left(\ell_{\infty} \cup Q(\nabla F)\right)$ and any incident line $\ell$ containing $m$, we define as follows the associated reflected line $\Re_{m}(\ell)$ (for the reflexion on $\mathcal{C}$ at $m$ with respect to the Snell-Descartes reflection law $\left.\operatorname{Angle}\left(\ell, \mathcal{T}_{m} \mathcal{C}\right)=\operatorname{Angle}\left(\mathcal{T}_{m} \mathcal{C}, \Re_{m}(\ell)\right)\right)$.

Definition 1.1. For every $m \in \mathcal{C} \backslash\left(\ell_{\infty} \cup V(Q(\nabla F))\right)$, we define $\mathfrak{r}_{m}: \ell_{\infty} \rightarrow \ell_{\infty}$ mapping $P \in \ell_{\infty}$ to the unique $\mathfrak{r}_{m}(P)$ such that $\beta\left(P, t_{m}, I, J\right)=\beta\left(t_{m}, \mathfrak{r}_{m}(P), I, J\right)$.

We define $\mathfrak{R}_{m}: \mathcal{F}_{m} \rightarrow \mathcal{F}_{m}$ with $\mathcal{F}_{m}:=\left\{\ell \in G\left(1, \mathbb{P}^{2}\right), m \in \ell\right\}$ by $\Re_{m}(\ell)=$ $\left(m \mathfrak{r}_{m}\left(P_{\ell}\right)\right)$ if $P_{\ell}$ is the point at infinity of $\ell$.

We have (on coordinates)

$$
\mathfrak{r}_{m}\left(\left[x_{1}: y_{1}: 0\right]\right)=\left[x_{1}\left(F_{x}^{2}-F_{y}^{2}\right)+2 y_{1} F_{x} F_{y}:-y_{1}\left(F_{x}^{2}-F_{y}^{2}\right)+2 x_{1} F_{x} F_{y}: 0\right] .
$$

Remark 1.2. Observe that $\mathfrak{r}_{m}$ is an involution on $\ell_{\infty} \cong \mathbb{P}^{1}$ with exactly two fixed points $t_{m}$ and $n_{m}\left[F_{x}: F_{y}: 0\right]$. As a consequence, $\Re_{m}$ is an involution with two fixed points $\mathcal{T}_{m}(\mathcal{C})$ and $\mathcal{N}_{m}(\mathcal{C}):=\left(m n_{m}\right)$ the normal line to $\mathcal{C}$ at $m$.

Moreover $\mathfrak{r}_{m}(I)=J$ and $\mathfrak{r}_{m}(J)=I$.

Definition 1.3. For any $m[x: y: z] \in \mathcal{C} \backslash\left(\{S\} \cup \ell_{\infty} \cup V(Q(\nabla F))\right.$ we define the reflected line $\mathcal{R}_{m}$ on $\mathcal{C}$ at $m$ (of the incident line coming from $S$ ) as the line $\mathcal{R}_{m}:=\Re_{m}((m S))$.

For $m[x: y: z] \in \mathcal{C} \backslash\left(\{S\} \cup \ell_{\infty} \cup V(Q(\nabla F))\right.$, the point at infinity of $(S m)$ is $s_{m}\left[x_{0} z-z_{0} x: y_{0} z-z_{0} y: 0\right]$. Due to the Euler identity, on $\mathcal{C}$, we have $x F_{x}+y F_{y}+z F_{z}=0$ and so $\left(x_{0} z-z_{0} x\right) F_{x}+\left(y_{0} z-z_{0} y\right) F_{y}=z_{\mathbf{S}} F$. Hence $\mathfrak{r}\left(s_{m}\right)=\left[-v_{\mathbf{m}}: u_{\mathbf{m}}: 0\right]$ and the reflected line $\mathcal{R}_{m}$ is the set of $P[X: Y: Z] \in \mathbb{P}^{2}$ such that $u_{\mathbf{m}} X+v_{\mathbf{m}} Y+w_{\mathbf{m}} Z=0$, with

$$
\begin{aligned}
u_{\mathbf{m}} & :=\left(z_{0} y-z y_{0}\right)\left(F_{x}^{2}+F_{y}^{2}\right)+2 z F_{y} \Delta_{\mathbf{S}} F \in \operatorname{Sym}^{2 d-1}\left(\mathbf{V}^{\vee}\right) \\
v_{\mathbf{m}} & :=\left(z x_{0}-z_{0} x\right)\left(F_{x}^{2}+F_{y}^{2}\right)-2 z F_{x} \Delta_{\mathbf{S}} F \in \operatorname{Sym}^{2 d-1}\left(\mathbf{V}^{\vee}\right) \\
w_{\mathbf{m}} & :=\frac{-x u_{m}-y v_{m}}{z}=\left(x y_{0}-y x_{0}\right)\left(F_{x}^{2}+F_{y}^{2}\right)-2 \Delta_{\mathbf{S}} F\left(x F_{y}-y F_{x}\right) \\
& \in \operatorname{Sym}^{2 d-1}\left(\mathbf{V}^{\vee}\right) .
\end{aligned}
$$

Definition 1.4. We call reflected map of $\mathcal{C}$ from $S$ the following rational map

$$
R_{\mathcal{C}, S}: \begin{aligned}
& \mathbb{P}^{2}-\rightarrow \\
& \left.m \mapsto u_{\mathbf{m}}: v_{\mathbf{m}}: w_{\mathbf{m}}\right]
\end{aligned} .
$$

We also define the rational map $T_{\mathcal{C}, S}:=\left(R_{\mathcal{C}, S}\right)_{\mid \mathcal{C}}: \mathcal{C} \rightarrow \mathbb{P}^{2}$ 
For any $\mathbf{m} \in \mathbf{V}$, it will be useful to define $\mathbf{R}_{F, \mathbf{S}}(\mathbf{m}):=\left(u_{\mathbf{m}}, v_{\mathbf{m}}, w_{\mathbf{m}}\right) \in \mathbf{V}$ and to notice that

$$
\mathbf{R}_{F, \mathbf{S}}(\mathbf{m})=Q(\nabla F(\mathbf{m})) \cdot(\mathbf{m} \wedge \mathbf{S})-2 \Delta_{\mathbf{S}} F(\mathbf{m}) \cdot\left(\mathbf{m} \wedge \mathbf{n}_{\mathbf{m}}\right) \in \mathbf{V}
$$

with $^{1} \mathbf{n}_{\mathbf{m}}\left(F_{x}(\mathbf{m}), F_{y}(\mathbf{m}), 0\right) \in \mathbf{V}$.

Proposition 1.5. The base points of $T_{\mathcal{C}, S}$ are the following: $I, J, S$ (if these points are in $\mathcal{C})$, the singular points of $\mathcal{C}$ and the points of tangency of $\mathcal{C}$ with some line of the triangle $(I J S)$.

Proof. We have to prove that the set of base points of $T_{\mathcal{C}, S}$ is the following set: $\mathcal{M}:=\mathcal{C} \cap\left(\{I, J, S\} \cup V\left(\Delta_{\mathbf{S}} F, Q(\nabla F)\right) \cup V\left(F_{x}, F_{y}\right)\right)$. We just prove that $\operatorname{Base}\left(T_{\mathcal{C}, S}\right) \subset$ $\mathcal{M}$, the converse being obvious (observe that if $m \in\{I, J\}$, we automatically have $Q(\nabla F(\mathbf{m}))=0$ and $\left.\mathbf{n}_{\mathbf{m}} \in \operatorname{Vect}(\mathbf{m})\right)$. Let $m[x ; y ; z] \in \mathcal{C}$ be such that $\mathbf{R}_{F, \mathbf{S}}(\mathbf{m})=\mathbf{0}$. Then $\mathbf{m}$ and $Q(\nabla F(\mathbf{m})) \cdot \mathbf{S}-2 \Delta_{\mathbf{S}} F(\mathbf{m}) \cdot \mathbf{n}_{\mathbf{m}}$ are colinear. Due to the Euler identity, we have $0=D F(\mathbf{m}) \cdot \mathbf{m}$ (with $D F(\mathbf{m})$ the differential of $F$ at $\mathbf{m})$ and so $0=-\Delta_{\mathbf{S}} F(\mathbf{m}) \cdot Q(\nabla F(\mathbf{m}))$ since $D F(\mathbf{m}) \cdot \mathbf{S}=\Delta_{\mathbf{S}} F(\mathbf{m})$ and since $D F(\mathbf{m}) \cdot \mathbf{n}_{\mathbf{m}}=Q(\nabla F(\mathbf{m}))$. Hence $\Delta_{\mathbf{S}} F(\mathbf{m})=0$ or $Q(\nabla F(\mathbf{m}))=0$.

If $\Delta_{\mathbf{S}} F(\mathbf{m})=0$, then either $Q(\nabla F(\mathbf{m}))=0$ or $m=S$.

If $\Delta_{\mathbf{S}} F(\mathbf{m}) \neq 0$ and $Q(\nabla F(\mathbf{m}))=0$, then $F_{x}(\mathbf{m})=F_{y}(\mathbf{m})=0$ or $m=$ $\left[F_{x}(\mathbf{m}): F_{y}(\mathbf{m}): 0\right]$. Assume that $m=\left[F_{x}(\mathbf{m}): F_{y}(\mathbf{m}): 0\right]$. Then, since $Q(\nabla F(\mathbf{m}))=0$, we conclude that $m \in\{I, J\}$.

We recall the following result established at the same period in [17] and by Catanese in [5], with two different proofs.

Proposition $1.6([5,17])$. Let $\mathcal{C}$ be an irreducible curve of degree $d \geq 2$. Then, for a generic $S \in \mathbb{P}^{3}$, the map $T_{\mathcal{C}, S}$ is birational.

\section{Caustic by reflection}

Definition 2.1. The caustic by reflection $\Sigma_{S}(\mathcal{C})$ is the Zariski closure of the envelope of the reflected lines $\left\{\mathcal{R}_{m} ; m \in \mathcal{C} \backslash\left(\{S\} \cup \ell_{\infty} \cup V(Q(\nabla F))\right\}\right.$.

Recall that, in [16], we have defined a rational map $\Phi_{F, \mathbf{S}}$ called caustic map mapping a generic $m \in \mathcal{C}$ to the point of tangency of $\Sigma_{S}(\mathcal{C})$ with $\mathcal{R}_{m}$ and that $\Sigma_{S}(\mathcal{C})$ is the Zariski closure of $\Phi_{F, \mathbf{S}}(\mathcal{C})$.

In the present work, we will not consider the cases in which the caustic by reflection $\Sigma_{S}(\mathcal{C})$ is a single point. We recall that these cases are easily characterized as follows.

Proposition 2.2. Assume that

(i) $S \notin\{I, J\}$,

${ }^{1}$ With $\wedge: \mathbf{V} \times \mathbf{V} \rightarrow \mathbf{V}$ being given in coordinates by $\left(x_{1}, y_{1}, z_{1}\right) \wedge\left(x_{2}, y_{2}, z_{2}\right)=\left(\begin{array}{l}z_{2} y_{1}-z_{1} y_{2} \\ z_{1} x_{2}-z_{2} x_{1} \\ x_{1} y_{2}-y_{1} x_{2}\end{array}\right)$. 
(ii) $\mathcal{C}$ is not a line (i.e. $d \neq 1)$,

(iii) if $d=2$, then $S$ is not a focus of the conic $\mathcal{C}$.

Then $\Sigma_{S}(\mathcal{C})$ is not reduced to a point and is an irreducible curve.

Proof. Assume (i), (ii) and (iii) and that $\Sigma_{S}(\mathcal{C})=\left\{S^{\prime}\right\}$ with $S^{\prime}=\left[x_{1}: y_{1}: z_{1}\right]$.

When $S \notin \ell_{\infty}$, we will use the fact that $\Sigma_{S}(\mathcal{C})$ is the evolute of the orthotomic of $\mathcal{C}$ with respect to $S$. Since $\mathcal{C}$ is not a line, the orthotomic of $\mathcal{C}$ with respect to $S$ is not reduced to a point but its evolute is a point. This implies that the orthotomic of $\mathcal{C}$ with respect to $S$ is either a line (not equal to $\ell_{\infty}$ ) or a circle. But $\mathcal{C}$ is the contrapedal (or orthocaustic) curve (from $S$ ) of the image by the $S$-centered homothety (with ratio $1 / 2$ ) of the orthotomic of $\mathcal{C}$. Therefore $d=2$ and $S$ is a focal point of $\mathcal{C}$, which contradicts (iii).

When $S \in \ell_{\infty}$ but $S^{\prime} \notin \ell_{\infty}$, then, for symmetry reasons, we also have $\Sigma_{S^{\prime}}(\mathcal{C})=\{S\}$ and we conclude analogously.

Suppose now that $S, S^{\prime} \in \ell_{\infty}$. We have $z_{0}=z_{1}=0$. For every $m=[x: y$ : $1] \in \mathcal{C} \backslash\left(\ell_{\infty} \cup V(Q(\nabla F))\right)$, we have $\beta\left(S, t_{m}, I, J\right)=\beta\left(t_{m}, S^{\prime}, I, J\right)$. Therefore we have

$$
\frac{\left(i x_{0}-y_{0}\right)\left(-i F_{y}+F_{x}\right)}{\left(i F_{y}+F_{x}\right)\left(-i x_{0}-y_{0}\right)}=\frac{\left(i F_{y}+F_{x}\right)\left(-i x_{1}-y_{1}\right)}{\left(-i F_{y}+F_{x}\right)\left(i x_{1}-y_{1}\right)}
$$

and so

$$
\left(i x_{0}-y_{0}\right)\left(i x_{1}-y_{1}\right)\left(-i F_{y}+F_{x}\right)^{2}=\left(i F_{y}+F_{x}\right)^{2}\left(-i x_{0}-y_{0}\right)\left(-i x_{1}-y_{1}\right) \text {. }
$$

Now, according to (i), $i x_{0}-y_{0} \neq 0,-i x_{0}-y_{0} \neq 0, i x_{1}-y_{1} \neq 0,-i x_{1}-y_{1} \neq 0$. Hence $\left(-i F_{y}+F_{x}\right)^{2}=a\left(i F_{y}+F_{x}\right)^{2}$ for some $a \neq 0$. So, there exists $(\alpha, \beta) \in$ $\mathbb{C}^{2} \backslash\{(0,0)\}$ such that $\alpha F_{x}+\beta F_{y}=0$ (setting $(\alpha, \beta)=\left(1-a_{0},-i-i a_{0}\right)$ for some $a_{0} \in \mathbb{C}$ satisfying $\left.a_{0}^{2}=a\right)$. This implies that $F(x, y, z)=G(\beta x-\alpha y, z)$ for some irreducible $G \in \operatorname{Sym}^{d}\left(\left(\mathbb{C}^{2}\right)^{\vee}\right)$. We conclude that $\operatorname{deg} G=1$ and so $d=1$ which contradicts (ii).

Hence we proved that $\Sigma_{S}(\mathcal{C})$ is not reduced to a point. Now the irreducibility of $\Sigma_{S}(\mathcal{C})$ comes from the fact that $\Sigma_{S}(\mathcal{C})=\overline{\Phi_{F, S}(\mathcal{C})}$ and that $\mathcal{C}$ is an irreducible curve.

Proposition 2.3. Assume that $\Sigma_{S}(\mathcal{C})$ is not reduced to a point. Then we have

$$
\operatorname{class}\left(\Sigma_{S}(\mathcal{C})\right)=\operatorname{deg}\left(\overline{T_{\mathcal{C}, S}(\mathcal{C})}\right)
$$

where $\overline{T_{\mathcal{C}, S}(\mathcal{C})}$ stands for the Zariski closure of $T_{\mathcal{C}, S}(\mathcal{C})$.

Proof. This comes from the fact that $\Sigma_{S}(\mathcal{C})$ is the Zariski closure of the envelope of $\left\{\mathcal{R}_{m}, m \in \mathcal{C} \backslash\left(\operatorname{Sing}(\mathcal{C}) \cup\{S\} \cup \ell_{\infty} \cup V(Q(\nabla F))\right\}\right.$ and can be precised as follows. For every algebraic curve $\Gamma=V(G)\left(\right.$ with $G$ in $\operatorname{Sym}^{k}\left(\mathbf{V}^{\vee}\right)$ for some $\left.k\right)$, 
we consider the Gauss map $\delta_{\Gamma}: \mathbb{P}^{2} \rightarrow-\rightarrow \mathbb{P}^{2}$ defined on coordinates by $\delta_{\Gamma}([x: y$ : $z])=\left[G_{x}: G_{y}: G_{z}\right]$, we obtain immediately the commutative diagram:

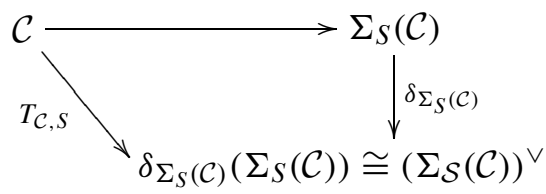

with $\Phi_{F, \mathbf{S}}$ the caustic map defined in [16] (see the begining of the present section).

Let us notice that, according to the proof of Proposition 2.3, the rational map $T_{\mathcal{C}, S}$ has the same degree as the rational map $\left(\Phi_{F, \mathbf{S}}\right)_{\mid \mathcal{C}}\left(\right.$ since $\Sigma_{S}(\mathcal{C})$ is irreducible and since the Gauss map $\left(\delta_{\Sigma_{S}(\mathcal{C})}\right)_{\mid \Sigma_{S}(\mathcal{C})}$ is birational [12]).

\section{Formulas for the class of the caustic}

Since the map $T_{\mathcal{C}, S}$ may be non birational, we introduce the notion of class with multiplicity of $\Sigma_{S}(\mathcal{C})$ :

$$
\operatorname{mclass}\left(\Sigma_{S}(\mathcal{C})\right)=\delta_{1}(S, \mathcal{C}) \times \operatorname{mclass}\left(\Sigma_{S}(\mathcal{C})\right)
$$

where class $\left(\Sigma_{S}(\mathcal{C})\right)$ is the class of the algebraic curve $\Sigma_{S}(\mathcal{C})$ and where $\delta_{1}(S, \mathcal{C})$ is the degree of the rational map $T_{\mathcal{C}, S}$. We recall that $\delta_{1}(S, \mathcal{C})$ corresponds to the number of preimages on $\mathcal{C}$ of a generic point of $\Sigma_{S}(\mathcal{C})$ by $T_{\mathcal{C}, S}$.

Before stating our main result, let us introduce some notations. For every $m_{1} \in \mathbb{P}^{2}$, we write $\mu_{m_{1}}=\mu_{m_{1}}(\mathcal{C})$ for the multiplicity of $m_{1}$ on $\mathcal{C}$ and consider the set $\operatorname{Branch}_{m_{1}}(\mathcal{C})$ of branches of $\mathcal{C}$ at $m_{1}$. We denote by $\mathcal{E}$ the set of couples pointbranch $\left(m_{1}, \mathcal{B}\right)$ of $\mathcal{C}$ with $m_{1} \in \mathcal{C}$ and $\mathcal{B} \in \operatorname{Branch}_{m_{1}}(\mathcal{C})$. For every $\left(m_{1}, \mathcal{B}\right) \in \mathcal{E}$, we write $e_{\mathcal{B}}$ for the multiplicity of $\mathcal{B}$ and $\mathcal{T}_{m_{1}}(\mathcal{B})$ the tangent line to $\mathcal{B}$ at $m_{1}$; we observe that $\mu_{m_{1}}=\sum_{\mathcal{B} \in \operatorname{Branch}_{m_{1}}(\mathcal{C})} e_{\mathcal{B}}$. We write $i_{m_{1}}\left(\Gamma, \Gamma^{\prime}\right)$ the intersection number of two curves $\Gamma$ and $\Gamma^{\prime}$ at $m_{1}$. For any algebraic curve $\mathcal{C}^{\prime}$ of $\mathcal{P}^{2}$, we also define the contact number $\Omega_{m_{1}}\left(\mathcal{C}, \mathcal{C}^{\prime}\right)$ of $\mathcal{C}$ and $\mathcal{C}^{\prime}$ at $m_{1} \in \mathbb{P}^{2}$ by

$$
\Omega_{m_{1}}\left(\mathcal{C}, \mathcal{C}^{\prime}\right):=i_{m_{1}}\left(\mathcal{C}, \mathcal{C}^{\prime}\right)-\mu_{m_{1}}(\mathcal{C}) \mu_{m_{1}}\left(\mathcal{C}^{\prime}\right) \quad \text { if } m_{1} \in \mathcal{C} \cap \mathcal{C}^{\prime}
$$

and

$$
\Omega_{m_{1}}\left(\mathcal{C}, \mathcal{C}^{\prime}\right):=0 \quad \text { if } m_{1} \notin \mathcal{C} \cap \mathcal{C}^{\prime} .
$$

Recall that $\Omega_{m_{1}}\left(\mathcal{C}, \mathcal{C}^{\prime}\right)=0$ means that $m_{1} \notin \mathcal{C} \cap \mathcal{C}^{\prime}$ or that $\mathcal{C}$ and $\mathcal{C}^{\prime}$ intersect transversally at $m_{1}$.

Theorem 3.1. Assume that the hypotheses of Proposition 2.2 hold. 
(1) If $S \notin \ell_{\infty}$, the class (with multiplicity) of $\Sigma_{S}(\mathcal{C})$ is given by

$$
\operatorname{mclass}\left(\Sigma_{S}(\mathcal{C})\right)=2 d^{\vee}+d-2 f^{\prime}-g-f-g^{\prime}+q^{\prime},
$$

where

- $g$ is the contact number of $\mathcal{C}$ with $\ell_{\infty}$, i.e. $g:=\sum_{m_{1} \in \mathcal{C} \cap \ell_{\infty}} \Omega_{m_{1}}\left(\mathcal{C}, \ell_{\infty}\right)$,

- $f$ is the multiplicity number at a cyclic point of $\mathcal{C}$ with an isotropic line from $S$, i.e.

$$
f:=i_{I}(\mathcal{C},(I S))+i_{J}(\mathcal{C},(J S)),
$$

- $f^{\prime}$ is the contact number of $\mathcal{C}$ with an isotropic line from $S$ outside $\{I, J, S\}$, i.e.

$$
f^{\prime}:=\sum_{m_{1} \in(\mathcal{C} \cap(I S)) \backslash\{I, S\}} \Omega_{m_{1}}(\mathcal{C},(I S))+\sum_{m_{1} \in(\mathcal{C} \cap(J S)) \backslash\{J, S\}} \Omega_{m_{1}}(\mathcal{C},(J S)),
$$

- $g^{\prime}$ given by $g^{\prime}:=i_{S}(\mathcal{C},(I S))+i_{S}(\mathcal{C},(J S))-\mu_{S}$;

- $q^{\prime}$ is given by

$$
q^{\prime}:=\sum_{\substack{\left(m_{1}, \mathcal{B}\right) \in \mathcal{E}: m_{1} \notin\{I, J, S\}, T_{m_{1}} \mathcal{B}=(I S) \\ \text { or } \\ T_{m_{1}} \mathcal{B}=(J S), i_{m_{1}}\left(\mathcal{B}, \mathcal{T}_{m_{1}}(\mathcal{B})\right) \geq 2 e_{\mathcal{B}}}}\left[i_{m_{1}}\left(\mathcal{B}, \mathcal{T}_{m_{1}}(\mathcal{B})\right)-2 e_{\mathcal{B}}\right] .
$$

(2) If $S \in \ell_{\infty}$, the class of $\Sigma_{S}(\mathcal{C})$ is

$$
\operatorname{mclass}\left(\Sigma_{S}(\mathcal{C})\right)=2 d^{\vee}+d-2 g-\mu_{I}-\mu_{J}-\mu_{S}-c^{\prime}(S),
$$

with

$$
c^{\prime}(S):=\sum_{\mathcal{B} \in \operatorname{Branch}_{S}(\mathcal{C}): i_{S}\left(\mathcal{B}, \ell_{\infty}\right)=2 e_{\mathcal{B}}}\left(e_{\mathcal{B}}+\left(\min \left(i_{S}\left(\mathcal{B}, O s c_{S}(\mathcal{B})\right)-3 e_{\mathcal{B}}, 0\right)\right),\right.
$$

where $\operatorname{Osc}_{S}(\mathcal{B})$ is any smooth algebraic osculating curve to $\mathcal{B}$ at $S$ (i.e. any smooth algebraic curve $\mathcal{C}^{\prime}$ such that $\left.i_{S}\left(\mathcal{B}, \mathcal{C}^{\prime}\right)>2 e_{\mathcal{B}}\right)$.

The notation introduced in this theorem is close to that of Salmon and Cayley [19] (see Section 5 for further explanations). Let us point out that, in this article, $g$ is not the geometric genus of the curve.

Remark 3.2. Observe that we also have

$$
c^{\prime}(S):=\sum_{\mathcal{B} \in \operatorname{Branch}_{S}(\mathcal{C}): i_{S}\left(\mathcal{B}, \ell_{\infty}\right)=2 e_{\mathcal{B}}}\left(e_{\mathcal{B}}+\min \left(\beta_{1}(S, \mathcal{B})-3 e_{\mathcal{B}}, 0\right)\right),
$$

where $\beta_{1}(S, \mathcal{B})$ is the first characteristic exponent of $\mathcal{B}$ which is not a multiple of $e_{\mathcal{B}}($ see $[25])$.

Observe that, when $i_{S}\left(\mathcal{B}, \mathcal{T}_{S}(\mathcal{B})\right)=2 e_{\mathcal{B}}$, we have $\min \left(i_{S}\left(\mathcal{B}, O s c_{S}(\mathcal{B})\right)-\right.$ $\left.3 e_{\mathcal{B}}, 0\right)=0$ except if $S$ is a singular point and if the probranches of $\mathcal{B}$ are given by $Y-x_{0}^{-1} y_{0}=\alpha Z^{2}+\alpha_{1} Z^{\beta_{1}}+\cdots$ in the chart $X=1$ if $x_{0} \neq 0$ (or $X-y_{0}^{-1} x_{0}=$ $\alpha Z^{2}+\alpha_{1} Z^{\beta_{1}}+\cdots$ in the chart $Y=1$ otherwise), with $\alpha \neq 0, \alpha_{1} \neq 0$ and $2<\beta_{1}<3$. Hence $c^{\prime}(S)=\sum_{\mathcal{B} \in \operatorname{Branch}_{S}(\mathcal{C}): i_{S}\left(\mathcal{B}, \ell_{\infty}\right)=2 e_{\mathcal{B}}} e_{\mathcal{B}}$ when $\mathcal{C}$ admits no such branch tangent at $S$ to $\ell_{\infty}$. 
Combining Proposition 1.6 and Theorem 3.1, we obtain:

Corollary 3.3 (A source-generic formula for the class). Let $\mathcal{C} \subset \mathbb{P}^{2}$ be a fixed curve of degree $d \geq 2$. For a generic source point $S$, we have $\delta_{1}(S, \mathcal{C})=1$ and $\operatorname{class}\left(\Sigma_{S}(\mathcal{C})\right)=2 \bar{d}^{\vee}+d-g-\mu_{I}-\mu_{J}$ where $g$ denotes the contact number of $\mathcal{C}$ with $\ell_{\infty}$.

Proof. Due to Proposition 1.6, $\delta_{1}(S, \mathcal{C})=1$ for a generic $S \in \mathbb{P}^{2}$. So $\operatorname{class}\left(\Sigma_{S}(\mathcal{C})\right)=$ $\operatorname{mclass}\left(\Sigma_{S}(\mathcal{C})\right)$.

Assume moreover, that $S \notin \ell_{\infty}$ (so we apply the first formula of Theorem 3.1), $S \notin \mathcal{C}$ (so $g^{\prime}=0$ ), that $(I S)$ and $(J S)$ are not tangent to $\mathcal{C}$ (so $f^{\prime}=q^{\prime}=0$ and $\left.f=\mu_{I}(\mathcal{C})+\mu_{J}(\mathcal{C})\right)$. We obtain the result.

\section{Examples}

Let us now illustrate our result for two particular mirror curves.

\subsection{Example of the lemniscate of Bernoulli}

We consider the case when $\mathcal{C}=V(F)$ is the lemniscate of Bernoulli given by $F(x, y, z)=\left(x^{2}+y^{2}\right)^{2}-2\left(x^{2}-y^{2}\right) z^{2}$ and when $S \in \mathbb{P}^{2} \backslash\{I, J\}$. The degree of $\mathcal{C}$ is $d=4$. The singular points of $\mathcal{C}$ are $: I[1: i: 0], J[1:-i: 0]$ and $\mathcal{O}[0: 0: 1]$. These three points are double points, each one having two different tangent lines. Hence the class of $\mathcal{C}$ is given by $d^{\vee}=d(d-1)-3 \times 2=6$ and so

$$
2 d^{\vee}+d=16 .
$$

The tangent lines to $\mathcal{C}$ at $I$ are $\ell_{1, I}:=V(y-i z-i x)$ and $\ell_{2, I}:=V(y-i z+i x)$ (the intersection number of $\mathcal{C}$ with $\ell_{1, I}$ or with $\ell_{2, I}$ at $I$ is equal to 4$)$. The tangent lines to $\mathcal{C}$ at $J$ are $\ell_{1, J}:=V(y+i z-i x)$ and $\ell_{2, J}:=V(y+i z+i x)$ (the intersection number of $\mathcal{C}$ with $\ell_{1, J}$ or with $\ell_{2, J}$ at $J$ is equal to 4 ). This ensures that we have

$$
f=2\left(2+\mathbf{1}_{S \in \ell_{1, I}}+\mathbf{1}_{S \in \ell_{2, I}}+\mathbf{1}_{S \in \ell_{1, J}}+\mathbf{1}_{S \in \ell_{2, J}}\right) .
$$

Observe that $\ell_{\infty}$ is not tangent to $\mathcal{C}$. Indeed $I$ and $J$ are the only points in $\mathcal{C} \cap \ell_{\infty}$ and $\ell_{\infty}$ is not tangent to $\mathcal{C}$ at these points. Therefore we have $g=0$ and $c^{\prime}(S)=0$.

Since $I$ and $J$ are also the only points at which $\mathcal{C}$ is tangent to an isotropic line (i.e. a line containing $I$ or $J$ ), we have $f^{\prime}=0, g^{\prime}=\mu_{S}, q^{\prime}=0$. In this case, one can check that $\delta_{1}(S, \mathcal{C})=1$. Finally, we get

$$
\text { if } S \notin \ell_{\infty}, \quad \operatorname{class}\left(\Sigma_{S}(\mathcal{C})\right)=12-2\left(\mathbf{1}_{S \in \ell_{1, I} \cup \ell_{2, I}}+\mathbf{1}_{S \in \ell_{1, J} \cup \ell_{2, J}}\right)-\mu_{S} .
$$

Moreover we have

$$
\text { if } S \in \ell_{\infty} \backslash\{I, J\}, \quad \operatorname{class}\left(\Sigma_{S}(\mathcal{C})\right)=16-2-2=12,
$$

(since $\mu_{I}=\mu_{J}=2$ and since $\mu_{S}=0$ ). For example, for $S[1: 0: 1]$, we get $\operatorname{class}\left(\Sigma_{S}(\mathcal{C})\right)=8$, since $S$ is in $\ell_{2, I} \cap \ell_{1, J}$ but not in $\mathcal{C}\left(\right.$ so $\left.\mu_{S}=0\right)$. 


\subsection{Example of a quintic curve}

As in [16], we consider the quintic curve $\mathcal{C}=V(F)$ with $F(x, y, z)=y^{2} z^{3}-x^{5}$. We also consider a light point $S\left[x_{0}: y_{0}: z_{0}\right] \in \mathbb{P}^{2} \backslash\{I, J\}$. This curve admits two singular points: $m_{1}[0: 0: 1]$ and $m_{2}[0: 1: 0]$, we have $d=5$.

We recall that $\mathcal{C}$ admits a single branch at $m_{1}$, which has multiplicity 2 and which is tangent to $V(y)$. We observe that $i_{m_{1}}(\mathcal{C}, V(y))=5$.

Analogously, $\mathcal{C}$ admits a single branch at $m_{2}$, which has multiplicity 3 and which is tangent to $\ell_{\infty}$. We observe that $i_{m_{2}}\left(\mathcal{C}, \ell_{\infty}\right)=5$.

We obtain that the class of $\mathcal{C}$ is $d^{\vee}=5$ and that $\mathcal{C}$ has no inflexion point (these two facts are proved in [16]). In particular, we get that $2 d^{\vee}+d=15$. $f=0$.

Since $m_{2}$ is the only point of $\mathcal{C} \cap \ell_{\infty}$, we get that $g=\Omega_{m_{2}}\left(\mathcal{C}, \ell_{\infty}\right)=2$ and

The curve $\mathcal{C}$ admits six (pairwise distinct) isotropic tangent lines other than $\ell_{\infty}: \ell_{1}, \ell_{2}$ and $\ell_{3}$ containing $I$ defined by

$$
\ell_{k}=V\left(i x-y+\frac{3 i}{25} \alpha^{k} \sqrt[3]{20} z\right), \text { where } \alpha:=e^{\frac{2 i \pi}{3}}
$$

for $k=1,2,3$ and $\ell_{4}, \ell_{5}$ and $\ell_{6}$ containing $J$ defined by

$$
\ell_{3+k}=V\left(i x+y+\frac{3 i}{25} \alpha^{k} \sqrt[3]{20} z\right)
$$

for $k=1,2,3$. For every $i \in\{1,2,3,4,5,6\}$, we write $a_{i}$ the point at which $\mathcal{C}$ is tangent to $\ell_{i}$ (the points $a_{i}$ correspond to the points of $\mathcal{C} \cap V\left(F_{x}^{2}+F_{y}^{2}\right) \backslash\left\{m_{1}, m_{2}\right\}$ ). Since $\mathcal{C}$ contains no inflexion point and since $m_{1}$ and $m_{2}$ are the only singular points of $\mathcal{C}$, we get that,

$$
f^{\prime}=\#\left\{i \in\{1,2,3,4,5,6\}: S \in \ell_{i} \backslash\left\{a_{i}\right\}\right\} \text { and } q^{\prime}=0
$$

when $S \notin \ell_{\infty}$.

Now recall that $g^{\prime}=i_{S}(\mathcal{C},(I S))+i_{S}(\mathcal{C},(J S))-\mu_{S}$. Again, in this case, one can check that $\delta_{1}(S, \mathcal{C})=1$. If $S \notin \ell_{\infty}$, we have

$$
\text { class }\left(\Sigma_{S}(\mathcal{C})\right)=13-2 \times \#\left\{i \in\{1,2,3,4,5,6\}: S \in \ell_{i} \backslash\left\{a_{i}\right\}\right\}-g^{\prime}
$$

and if $S \in \ell_{\infty} \backslash\{I, J\}$, we have

$$
\operatorname{class}\left(\Sigma_{S}(\mathcal{C})\right)=11-3 \times \mathbf{1}_{S=m_{2}} .
$$

We observe that the points of $\mathbb{P}^{2} \backslash\{I, J\}$ belonging to two dictinct $\ell_{k}$ are outside $\mathcal{C}$. The set of these points is

$$
\begin{gathered}
\mathcal{E}:=\bigcup_{k=1}^{3}\left\{\left[-\frac{3}{25} \sqrt[3]{20} \alpha^{k}: 0: 1\right],\left[\frac{3}{50} \sqrt[3]{20} \alpha^{k}: \frac{3}{50} \sqrt{3} \sqrt[3]{20} \alpha^{k}: 1\right],\right. \\
\left.\left[\frac{3}{50} \sqrt[3]{20} \alpha^{k}:-\frac{3}{50} \sqrt{3} \sqrt[3]{20} \alpha^{k}: 1\right]\right\}
\end{gathered}
$$


with $\alpha=e^{\frac{2 i \pi}{3}}$. Finally, the class of the caustic in the different cases is summarized in the following table.

\begin{tabular}{cc}
\hline Condition on $\boldsymbol{S} \in \mathbb{P}^{2} \backslash\{\boldsymbol{I}, \boldsymbol{J}\}$ & $\operatorname{class}\left(\boldsymbol{\Sigma}_{\boldsymbol{S}}(\mathcal{C})\right)=$ \\
\hline$S=m_{2}$ & 8 \\
$S \in \mathcal{E}$ & 9 \\
$S \in \mathcal{C} \cap \bigcup_{k=1}^{6}\left(\ell_{k} \backslash\left\{a_{k}\right\}\right)$ & 10 \\
$S \in\left(\ell_{\infty} \backslash\left\{m_{2}\right\}\right) \cup\left(\bigcup_{k=1}^{6} \ell_{k} \backslash(\mathcal{E} \cup \mathcal{C})\right) \cup\left\{m_{1}\right\} \cup\left\{a_{1}, \ldots, a_{6}\right\}$ & 11 \\
$S \in \mathcal{C} \backslash\left(\ell_{\infty} \cup\left\{m_{1}\right\} \cup \bigcup_{k=1}^{6} \ell_{k}\right)$ & 12 \\
otherwise & 13 \\
\hline
\end{tabular}

\section{On the formulas by Brocard and Lemoyne and by Salmon and Cayley}

\subsection{Formulas given by Brocard and Lemoyne}

Recall that, when $S \notin \ell_{\infty}, \Sigma_{S}(\mathcal{C})$ is the evolute of an homothetic of the pedal of $\mathcal{C}$ from $S$.

The work of Salmon and Cayley is under ordinary Plücker conditions (no worse multiple tangents than ordinary double tangents, no singularities other than ordinary cusps and ordinary nodes). In [19, page 137], Salmon and Cayley gave the following formula for the class of the evolute :

$$
n^{\prime}=m+n-f-g .
$$

Replace now $m, n, f$ and $g$ by $M, N, F$ and $G$ (respectively) given in [19, page 154] for the pedal. Doing so, one gets precisely (with the same notations) the formula of the class of caustics by reflection given by Brocard and Lemoyne in [1, page 114].

As explained in introduction, this composition of formulas of Salmon and Cayley is incorrect because of the non-conservation of the Plücker conditions by the pedal transformation. Nevertheless, for completeness sake, let us present the Brocard and Lemoyne formula and compare it with our formula. Brocard and Lemoyne gave the following formula for the class of the caustic by reflection $\Sigma_{S}(\mathcal{C})$ when $S \notin \ell_{\infty}$ :

$$
\mathrm{BL}=d+2\left(d^{\vee}-\hat{f}^{\prime}\right)-\hat{g}-\hat{f}-\hat{g}^{\prime}+\hat{q}^{\prime},
$$

for an algebraic curve $\mathcal{C}$ of degree $d$, of class $d^{\vee}, \hat{g}$ times tangent to $\ell_{\infty}$, passing $\hat{f}$ times through a cyclic point, $\hat{f}^{\prime}$ times tangent to an isotropic line of $S$, passing $\hat{g}^{\prime}$ times through $S, \hat{q}^{\prime}$ being the coincidence number of contact points when an 
isotropic line is multiply tangent. In [19], $\hat{q}^{\prime}$ is defined as the coincidence number of tangents at points $\iota_{1}, \iota_{2}$ of $\mathbb{P}^{2} \vee$ (corresponding to $(I S)$ and $(J S)$ ) if these points are multiple points of the image of $\mathcal{C}$ by the polar reciprocal transformation with center $S$; i.e. $\hat{q}^{\prime}$ represents the number of ordinary flexes of $\mathcal{C}$.

When $S \notin \ell_{\infty}$, let us compare terms appearing in our formula (3.1) with terms of (5.1):

- $\hat{g}$ seems to be equal to $g$;

- it seems that $\hat{f}=\mu_{I}+\mu_{J}$ and so $f=\hat{f}+\Omega_{I}(\mathcal{C},(I S))+\Omega_{J}(\mathcal{C},(J S))$;

- it seems that $\hat{f}^{\prime}=\sum_{m_{1} \in \mathcal{C} \cap(I S)} \Omega_{m_{1}}(\mathcal{C},(I S))+\sum_{m_{1} \in \mathcal{C} \cap(J S)} \Omega_{m_{1}}(\mathcal{C},(J S))$ and so

$$
f^{\prime}=\hat{f}^{\prime}-\Omega_{I}(\mathcal{C},(I S))-\Omega_{J}(\mathcal{C},(J S))-\Omega_{S}(\mathcal{C},(I S))-\Omega_{S}(\mathcal{C},(J S)) ;
$$

- it seems that $\hat{g}^{\prime}=\mu_{S}$, therefore $g^{\prime}=\hat{g}^{\prime}+\Omega_{S}(\mathcal{C},(I S))+\Omega_{S}(\mathcal{C},(J S))$;

- our definition of $q^{\prime}$ appears as an extension of $\hat{q}^{\prime}$ (except that we exclude the points $\left.m_{1} \in\{I, J, S\}\right)$.

Observe that these terms coincide with the definition of Brocard and Lemoyne if $(I S)$ and $(J S)$ are not tangent to $\mathcal{C}$ at $S, I, J$. In particular, the first item of Theorem 3.1 states that, when $S$ is not at infinity we have

$\operatorname{mclass}\left(\Sigma_{S}(\mathcal{C})\right)=\mathrm{BL}+\Omega_{I}(\mathcal{C},(I S))+\Omega_{J}(\mathcal{C},(J S))+\Omega_{S}(\mathcal{C},(I S))+\Omega_{S}(\mathcal{C},(J S))$, with BL the Brocard and Lemoyne formula recalled in (5.1). This last formula point out the error in the Brocard and Lemoyne formula.

\subsection{A counterexample to the formula of Brocard and Lemoyne}

We consider an example in which $\Omega_{I}(\mathcal{C},(I S))=\Omega_{J}(\mathcal{C},(J S))=1$, which means that $(I S)$ is tangent to $\mathcal{C}$ at $I$ and $(J S)$ is tangent to $\mathcal{C}$ at $J$. Let us consider the nonsingular quartic curve $\mathcal{C}=V\left(2 y z^{3}+2 z^{2} y^{2}+2 z y^{3}+2 y^{4}-2 z^{3} x+2 z y x^{2}+5 y^{2} x^{2}+\right.$ $3 x^{4}$ ) and $S[0: 0: 1]$. This curve $\mathcal{C}$ has degree $d=4$ and class $d^{\vee}=4 \times 3=12$, is not tangent to $\ell_{\infty}$, is tangent to $(S I)$ at $I$ and nowhere else, is tangent to $(S J)$ at $J$ and nowhere else; these tangent points are ordinary. $S$ is a non singular point of $\mathcal{C}$. Therefore, with our definitions, we have $g=0, f=2+2=4, f^{\prime}=0, g^{\prime}=$ $1+1-1=1, q^{\prime}=0$, which gives class $\left(\Sigma_{S}(\mathcal{C})\right)=4+2(12-0)-0-4-1-0=23$, since in this case $\delta_{1}(S, \mathcal{C})=1$. In comparison, the Brocard and Lemoyne formula would give $\hat{g}=0, \hat{f}=1+1=2, \hat{f}^{\prime}=1+1=2, \hat{g}^{\prime}=1, \hat{q}^{\prime}=0$ and so their formula gives $B L=4+2(12-2)-0-2-1-0=21$ which is not $\operatorname{class}\left(\Sigma_{S}(\mathcal{C})\right)$.

\section{Proof of Theorem 3.1}

To compute the degree of $\overline{T_{\mathcal{C}, S}(\mathcal{C})}$, we will use the Fundamental Lemma given in [16]. Let us first recall the definition of $\varphi$-polar introduced in [16] and extending the notion of polar. 
Definition 6.1. Let $p \geq 1, q \geq 1$ and let $\mathbf{W}$ be a complex vector space of dimension $p+1$. Given $\varphi: \mathbb{P}^{p}:=\mathbb{P}(\mathbf{W}) \rightarrow \mathbb{P}^{q}$ a rational map defined by $\varphi=\left[\varphi_{0}: \cdots: \varphi_{q}\right]\left(\right.$ with $\left.\varphi_{0}, \ldots, \varphi_{q} \in \operatorname{Sym}^{d}\left(\mathbf{W}^{\vee}\right)\right)$ and $a=\left[a_{0}: \cdots: a_{q}\right] \in \mathbb{P}^{q}$, we define the $\varphi$-polar at $a$, denoted by $\mathcal{P}_{\varphi, a}$, the hypersurface of degree $d$ given by $\mathcal{P}_{\varphi, a}:=V\left(\sum_{j=0}^{q} a_{j} \varphi_{j}\right) \subseteq \mathbb{P}^{p}$.

With this definition, the "classical" polar of a curve $\mathcal{C}=V(F)$ of $\mathbb{P}^{2}$ (for some homogeneous polynomial $F \in \mathbb{C}[x, y, z])$ at $a$ is the $\delta_{\mathcal{C}}$-polar curve at $a$, where $\delta_{\mathcal{C}}:[x: y: z] \mapsto\left[F_{x}: F_{y}: F_{z}\right]$.

Definition 6.2. We call reflected polar (or $r$-polar) of the plane curve $\mathcal{C}$ with

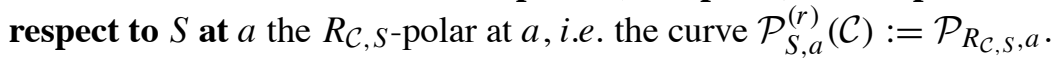

From a geometric point of view, $\mathcal{P}_{S, a}^{(r)}(\mathcal{C})$ is an algebraic curve such that, for every $m \in \mathcal{C} \cap \mathcal{P}_{S, a}^{(r)}(\mathcal{C}), \mathcal{R}_{m}$ contains $a$ (if $\mathcal{R}_{m}$ is well defined), this means that line (am) is tangent to $\Sigma_{S}(\mathcal{C})$ at the point $m^{\prime}=\Phi_{F, \mathbf{S}}(m) \in \Sigma_{S}(\mathcal{C})$ associated to $m$ (see picture).

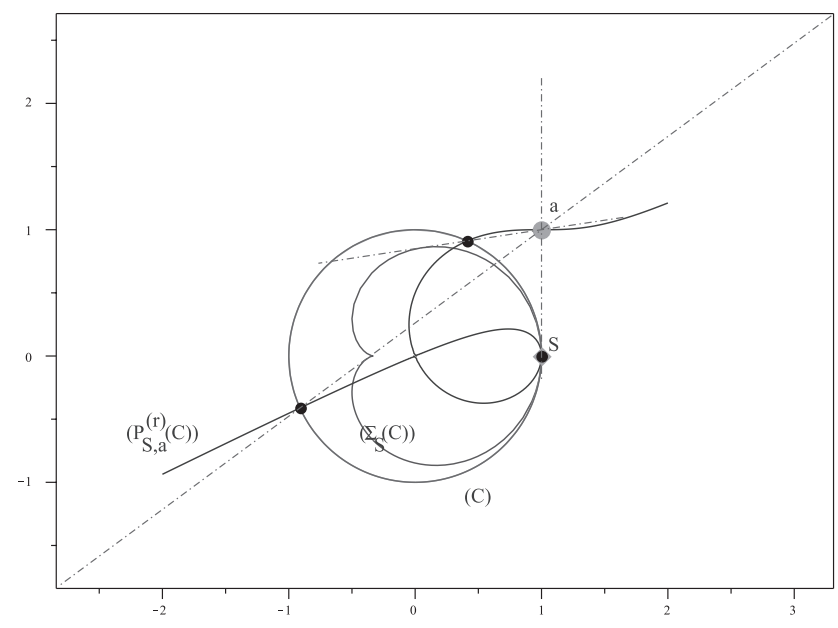

Let us now recall the statement of the fundamental lemma proved in [16].

Lemma 6.3 (Fundamental lemma [16]). Let $\mathbf{W}$ be a complex vector space of dimension $p+1$, let $\mathcal{C}$ be an irreducible algebraic curve of $\mathbb{P} p:=\mathbb{P}(\mathbf{W})$ and $\varphi: \mathbb{P}^{p} \rightarrow \mathbb{P}^{q}$ be a rational map given by $\varphi=\left[\varphi_{0}: \cdots: \varphi_{q}\right]$ with $\varphi_{0}, \ldots, \varphi_{q} \in$ $\operatorname{Sym}^{\delta}\left(\mathbf{W}^{\vee}\right)$. Assume that $\mathcal{C} \nsubseteq \operatorname{Base}(\varphi)$ and that $\varphi_{\mid \mathcal{C}}$ has degree $\delta_{1} \in \mathbb{N} \cup\{\infty\}$. Then, for generic $a=\left[a_{0}: \cdots: a_{q}\right] \in \mathbb{P}^{q}$, the following formula holds

$$
\delta_{1} \cdot \operatorname{deg}(\overline{\varphi(\mathcal{C})})=\delta \cdot \operatorname{deg}(\mathcal{C})-\sum_{p \in \operatorname{Base}(\varphi \mid \mathcal{C})} i_{p}\left(\mathcal{C}, \mathcal{P}_{\varphi, a}\right)
$$

with convention $0 . \infty=0$ and $\operatorname{deg}(\overline{\varphi(\mathcal{C})})=0$ if $\overline{\#(\mathcal{C})}<\infty$. 
Due to this lemma and to Proposition 2.3, we have

$$
\operatorname{mclass}\left(\Sigma_{S}(\mathcal{C})\right)=d(2 d-1)-\sum_{m_{1} \in \operatorname{Base}\left(T_{\mathcal{C}, S}\right)} i_{m_{1}}\left(\mathcal{C}, \mathcal{P}_{S, a}^{(r)}(\mathcal{C})\right)
$$

Now, we enter in the most technical stuff which is the computation of the intersection numbers $i_{m_{1}}\left(\mathcal{C}, \mathcal{P}_{S, a}^{(r)}(\mathcal{C})\right)$ of $\mathcal{C}$ with its reflected polar at the base points of $R_{\mathcal{C}, S}$. To compute these intersection numbers, it will be useful to observe the form of the image of $R_{\mathcal{C}, S}$ by linear changes of variable. It is worth noting that $\mathbf{R}_{F, \mathbf{S}}$ can be rewritten

$$
\mathbf{R}_{F, \mathbf{S}}=i d \wedge\left[\Delta_{\mathbf{I}} F \Delta_{\mathbf{J}} F \cdot \mathbf{S}-\Delta_{\mathbf{S}} F \Delta_{\mathbf{I}} F \cdot \mathbf{J}-\Delta_{\mathbf{S}} F \Delta_{\mathbf{J}} F \cdot \mathbf{I}\right] .
$$

Proposition 6.4. Let $M \in G L(\mathbf{V})$. We have

$$
\mathbf{R}_{F, \mathbf{S}} \circ M=\operatorname{Com}(M) \cdot \mathbf{R}_{F \circ M, M^{-1}(\mathbf{S})}^{\left(M^{-1}(\mathbf{I}), M^{-1}(\mathbf{J})\right)},
$$

with $\operatorname{Com}(M):=\operatorname{det}(M) \cdot{ }^{t} M^{-1}$ and

$$
\mathbf{R}_{G, \mathbf{S}^{\prime}}^{(\mathbf{A}, \mathbf{B})}:=i d \wedge\left[\Delta_{\mathbf{A}} G \Delta_{\mathbf{B}} G \cdot \mathbf{S}^{\prime}-\Delta_{\mathbf{S}^{\prime}} G \Delta_{\mathbf{A}} G \cdot \mathbf{B}-\Delta_{\mathbf{S}^{\prime}} G \Delta_{\mathbf{B}} G \cdot \mathbf{A}\right] .
$$

Proof. We use $M(\mathbf{u}) \wedge M(\mathbf{v})=(\operatorname{Com}(M))(\mathbf{u} \wedge \mathbf{v})$ and $\Delta_{M(\mathbf{u})}(F)(M(\mathbf{P}))=\Delta_{\mathbf{u}}(F \circ$ $M)(\mathbf{P})$.

We write $\Pi: \mathbf{V} \backslash\{\mathbf{0}\} \rightarrow \mathbb{P}^{2}$ for the canonical projection, $P_{0}[0: 0: 1] \in \mathbb{P}^{2}$ and $\mathbf{P}_{0}(0,0,1) \in \mathbf{V}$. Let $m_{1}$ be a base point of $\mathcal{C}$ and $M \in G L(\mathbf{V})$ be such that $\Pi\left(M\left(\mathbf{P}_{0}\right)\right)=m_{1}$ and such that the tangent cone of $V(F \circ M)$ at $P_{0}$ does not contain $V(x)$. Let $\mu_{m_{1}}$ be the multiplicity of $m_{1}$ in $\mathcal{C}\left(m_{1}\right.$ is a singular point of $\mathcal{C}$ if and only if $\left.\mu_{m_{1}}>1\right)$. Then, for every $a \in \mathbb{P}^{2}$, writing $a^{\prime}:=M^{-1}(a)$, we have

$$
\begin{aligned}
& i_{m_{1}}\left(\mathcal{C}, \mathcal{P}_{S, a}^{(r)}(\mathcal{C})\right)=i_{m_{1}}\left(\mathcal{C}, V\left(\left|\mathbf{a}, \mathbf{R}_{F, \mathbf{S}}(\cdot)\right\rangle\right)\right) \\
& =i_{P_{0}}\left(V(F \circ M), V\left(\left\langle\mathbf{a}, \mathbf{R}_{F, \mathbf{S}} \circ M(\cdot)\right\rangle\right)\right) \\
& =i_{P_{0}}\left(V(F \circ M), V\left(\left\langle\mathbf{a}^{\prime}, \mathbf{R}_{F \circ M, M^{-1}(\mathbf{S})}^{\left(M^{-1}(\mathbf{I}), M^{-1}(\mathbf{J})\right)}(\cdot)\right\rangle\right)\right) \\
& =\sum_{\mathcal{B} \in \operatorname{Branch}_{P_{0}}(V(F \circ M))} i_{P_{0}}\left(\mathcal{B}, V\left(\left\langle\mathbf{a}^{\prime}, \mathbf{R}_{F \circ M, M^{-1}(\mathbf{S})}^{\left(M^{-1}(\mathbf{I}), M^{-1}(\mathbf{J})\right)}(\cdot)\right\rangle\right)\right) \text {, }
\end{aligned}
$$

where $\operatorname{Branch}_{P_{0}}(V(F \circ M))$ is the set of branches of $V(F \circ M)$ at $P_{0}$. The last equality comes from Proposition A.1 proved in appendix (see formula (A.1)). Let $b$ be the number of such branches. Of course, $b=1$ for non-singular points. Writing $e_{\mathcal{B}}$ for the multiplicity of the branch $\mathcal{B}$, we have $\mu_{m_{1}}=\sum_{\mathcal{B} \in \operatorname{Branch}_{P_{0}}(V(F \circ M))} e_{\mathcal{B}}$. Let us write $\mathbb{C}\left\langle x^{\frac{1}{N}}\right\rangle$ and $\mathbb{C}\left\langle x^{\frac{1}{N}}, y\right\rangle$ for the rings of convergent power series of $x^{\frac{1}{N}}, y$. 
Let $\mathbb{C}\left\langle x^{*}\right\rangle:=\bigcup_{N \geq 1} \mathbb{C}\left\langle x^{\frac{1}{N}}\right\rangle$ and $\mathbb{C}\left\langle x^{*}, y\right\rangle:=\bigcup_{N \geq 1} \mathbb{C}\left\langle x^{\frac{1}{N}}, y\right\rangle$. For every $h=$ $\sum_{q \in \mathbb{Q}_{+}} a_{q} x^{q} \in \mathbb{C}\left\langle x^{*}\right\rangle$, we define the valuation of $h$ as follows:

$$
\operatorname{val}(h):=\operatorname{val}_{x}(h(x)):=\min \left\{q \in \mathbb{Q}_{+}, a_{q} \neq 0\right\} .
$$

Let $\mathcal{B}$ be a branch of $V(F \circ M)$ at $P_{0}$. Observe that $\mathcal{B}_{0}=M(\mathcal{B}) \subset \mathbb{P}^{2}$ is a branch of $\mathcal{C}$ at $m_{1}$. Let $\mathbf{A}\left(x_{A}, y_{A}, z_{A}\right):=M^{-1}(\mathbf{I}), \mathbf{B}\left(x_{B}, y_{B}, z_{B}\right):=M^{-1}(\mathbf{J})$ and $\mathbf{S}^{\prime}:=M^{-1}(\mathbf{S})$. Let $\mathcal{T}_{\mathcal{B}}$ be the tangent line to $\mathcal{B}$ at $P_{0}$. The branch $\mathcal{B}$ can be split in $e_{\mathcal{B}}$ pro-branches with equations $y=g_{i, \mathcal{B}}(x)$ in the chart $z=1$ (for $i \in\left\{1, \ldots, e_{\mathcal{B}}\right\}$ ) with $g_{i} \in \mathbb{C}\left\langle x^{*}\right\rangle$ having (rational) valuation larger than or equal to 1 (so $g_{i}^{\prime}(0)=0$ ). For $j \in\left\{1, \ldots, e_{\mathcal{B}^{\prime}}\right\}$, consider also the equations $y=g_{j, \mathcal{B}^{\prime}}(x)$ (in the chart $z=1$ ) of the pro-branches $\mathcal{V}_{j, \mathcal{B}^{\prime}}$ for each branch $\mathcal{B}^{\prime} \in \operatorname{Branch}_{P_{0}}(V(F \circ M))$. This notion of pro-branches comes from the combination of the Weierstrass and the Puiseux theorems. It has been used namely by Halphen in [14] and by Wall in [24]. One can also see [16]. There exists a unit $U$ in $\mathbb{C}\langle x, y\rangle$ such that the following equality holds in $\mathbb{C}\left\langle x^{*}, y\right\rangle$

$$
F(M(x, y, 1))=U(x, y) \prod_{\mathcal{B}^{\prime} \in \operatorname{Branch}_{P_{0}}(V(F \circ M))} \prod_{j=1}^{e_{\mathcal{B}^{\prime}}}\left(y-g_{j, \mathcal{B}^{\prime}}(x)\right) .
$$

For a generic $a$ (with $a^{\prime}:=M^{-1}(a)$ ), using (A.2)), we obtain

$$
\begin{aligned}
i_{P_{0}}\left(\mathcal{B}, V\left(\left\langle\mathbf{a}^{\prime}, \mathbf{R}_{F \circ M, \mathbf{S}^{\prime}}^{(\mathbf{A}, \mathbf{B})}(\cdot)\right\rangle\right)\right) & =\sum_{i} \operatorname{val}_{x}\left(\left\langle\mathbf{a}^{\prime}, \mathbf{R}_{F \circ M, \mathbf{S}^{\prime}}^{(\mathbf{A}, \mathbf{B})}\left(x, g_{i, \mathcal{B}}(x), 1\right)\right\rangle\right) \\
& =\sum_{i} \min _{j=1,2,3} \operatorname{val}_{x}\left(\left[\mathbf{R}_{F \circ M, \mathbf{S}^{\prime}}^{(\mathbf{A}, \mathbf{B})}\left(x, g_{i, \mathcal{B}}(x), 1\right)\right]_{j}\right) .
\end{aligned}
$$

Hence formula (6.1) becomes

$$
\begin{aligned}
\operatorname{mclass}\left(\Sigma_{S}(\mathcal{C})\right):= & d(2 d-1) \\
& -\sum_{m_{1} \in \mathcal{C}} \sum_{\mathcal{B}} \sum_{i=1}^{e_{\mathcal{B}}} \min _{j=1,2,3} \operatorname{val}_{x}\left(\left[\mathbf{R}_{\mathbf{F} \circ \mathbf{M}, \mathbf{S}^{\prime}}^{(\mathbf{A}, \mathbf{B})}\left(\mathbf{x}, \mathbf{g}_{\mathbf{i}, \mathcal{B}}(\mathbf{x}), \mathbf{1}\right)\right]_{j}\right),
\end{aligned}
$$

where, for every $m_{1} \in \mathcal{C}, M$ depends on $m_{1}$ and is as above, where the sum is over $\mathcal{B} \in \operatorname{Branch}_{P_{0}}(V(F \circ M))$. Due to Lemma 33 of [16], for every $\mathbf{P}\left(x_{P}, y_{P}, z_{P}\right) \in$ $\mathbf{V} \backslash\{\mathbf{0}\}$, we have

$$
\left(\Delta_{M(\mathbf{P})} F\right) \circ M\left(x, g_{i, \mathcal{B}}(x), 1\right)=\Delta_{\mathbf{P}}(F \circ M)\left(x, g_{i, \mathcal{B}}(x), 1\right)=D_{i, \mathcal{B}}(x) W_{\mathbf{P}, i, \mathcal{B}}(x),
$$

with

$$
W_{\mathbf{P}, i, \mathcal{B}}(x):=y_{P}-g_{i, \mathcal{B}}^{\prime}(x) x_{P}+z_{P}\left(x g_{i, \mathcal{B}}^{\prime}(x)-g_{i, \mathcal{B}}(x)\right)
$$

and with

$$
D_{i, \mathcal{B}}(x):=U\left(x, g_{i, \mathcal{B}}(x)\right) \prod_{\mathcal{B}^{\prime} \in \operatorname{Branch}_{P_{0}}(V(F \circ M))} \prod_{j=1, \ldots, e_{\mathcal{B}^{\prime}}:\left(\mathcal{B}^{\prime}, j\right) \neq(\mathcal{B}, i)}\left(g_{i, \mathcal{B}}(x)-g_{j, \mathcal{B}^{\prime}}(x)\right) .
$$


Hence we have

$$
\mathbf{R}_{F \circ M, \mathbf{S}^{\prime}}^{(\mathbf{A}, \mathbf{B})}\left(x, g_{i, \mathcal{B}}(x), 1\right):=\left(D_{i, \mathcal{B}}\right)^{2} \cdot \hat{R}_{i, \mathcal{B}}(x)
$$

with

$$
\begin{aligned}
\hat{R}_{i, \mathcal{B}}(x):= & \left(\begin{array}{c}
x \\
g_{i, \mathcal{B}}(x) \\
1
\end{array}\right) \wedge\left[W_{\mathbf{A}, i, \mathcal{B}}(x) W_{\mathbf{B}, i, \mathcal{B}}(x) \cdot \mathbf{S}^{\prime}-W_{\mathbf{S}^{\prime}, i, \mathcal{B}}(x) W_{\mathbf{A}, i, \mathcal{B}}(x) \cdot \mathbf{B}\right. \\
& \left.-W_{\mathbf{S}^{\prime}, i, \mathcal{B}}(x) W_{\mathbf{B}, i, \mathcal{B}}(x) \cdot \mathbf{A}\right] .
\end{aligned}
$$

First, with the notations of [16] (since $U(0,0) \neq 0$ ), we have

$$
\sum_{\mathcal{B} \in \operatorname{Branch}_{P_{0}}(V(F \circ M))} \sum_{i=1}^{e_{\mathcal{B}}} \operatorname{val}\left(D_{i, \mathcal{B}}\right)=V_{m_{1}}
$$

(which is zero if $m_{1}$ is a nonsingular point of $\mathcal{C}$ ). Second, writing $h_{m_{1}, i, \mathcal{B}}:=$ $\min \left(\operatorname{val}\left(\left[\hat{R}_{i, \mathcal{B}}\right]_{j}\right), j=1,2,3\right)$, we observe that, due to Proposition 29 and to Remark 34 of [16], the quantity $\sum_{i=1}^{e_{\mathcal{B}}} h_{m_{1}, i, \mathcal{B}}$ only depends on $m_{1}$ and on the branch $\mathcal{B}_{0}=M(\mathcal{B})$ of $\mathcal{C}$ at $m_{1}$ (it does not depend on the choice of $M \in G L(\mathbf{V})$ such that $\Pi\left(M\left(P_{0}\right)\right)=m_{1}$ and such that $V(x)$ is not tangent to $\left.M^{-1}\left(\mathcal{B}_{0}\right)\right)$. Hence we write

$$
h_{m_{1}, \mathcal{B}_{0}}:=\sum_{i=1}^{e_{\mathcal{B}}} h_{m_{1}, i, \mathcal{B}}
$$

With these notations, due to (6.3), formula (6.2) becomes

$$
\operatorname{mclass}\left(\Sigma_{S}(\mathcal{C})\right)=2 d(d-1)+d-2 \sum_{m_{1} \in \operatorname{Sing}(\mathcal{C})} V_{m_{1}}-\sum_{m_{1} \in \mathcal{C}} \sum_{\mathcal{B}_{0} \in \operatorname{Branch}_{m_{1}}(\mathcal{C})} h_{m_{1}, \mathcal{B}_{0}} .
$$

Moreover, as noticed in [16], we have $d(d-1)-\sum_{m_{1} \in \operatorname{Sing}(\mathcal{C})} V_{m_{1}}=d^{\vee}$, where $d^{\vee}$ is the class of $\mathcal{C}$. Therefore, we get

$$
\operatorname{mclass}\left(\Sigma_{S}(\mathcal{C})\right)=2 d^{\vee}+d-\sum_{m_{1} \in \mathcal{C}} \sum_{\mathcal{B}_{0} \in \operatorname{Branch}_{m_{1}}(\mathcal{C})} h_{m_{1}, \mathcal{B}_{0}} .
$$

Theorem 3.1 will come directly from the computation of $h_{m_{1}, i, \mathcal{B}}$ given in the following result.

Lemma 6.5. Let $m_{1} \in \mathcal{C}$ and $\mathcal{B}_{0} \in \operatorname{Branch}_{m_{1}}(\mathcal{C})$. Writing $\mathcal{T}_{m_{1}} \mathcal{B}_{0}$ for the tangent line to $\mathcal{B}_{0}$ at $m_{1}, i_{m_{1}}\left(\mathcal{B}_{0}, \mathcal{T}_{m_{1}} \mathcal{B}_{0}\right)$ for the intersection number of $\mathcal{B}_{0}$ with $\mathcal{T}_{m_{1}} \mathcal{B}_{0}$ at $m_{1}$ and $e_{\mathcal{B}_{0}}$ for the multiplicity of $\mathcal{B}_{0}$, we have

(1) $h_{m_{1}, \mathcal{B}_{0}}=0$ if $I, J, S \notin \mathcal{T}_{m_{1}} \mathcal{B}_{0}$.

(2) $h_{m_{1}, \mathcal{B}_{0}}=0$ if $\#\left(\mathcal{T}_{m_{1}} \mathcal{B}_{0} \cap\{I, J, S\}\right)=1$ and $m_{1} \notin\{I, J, S\}$. 
(3) $h_{m_{1}, \mathcal{B}_{0}}=e_{\mathcal{B}_{0}}$ if $\#\left(\mathcal{T}_{m_{1}} \mathcal{B}_{0} \cap\{I, J, S\}\right)=1$ and $m_{1} \in\{I, J, S\}$.

(4) $h_{m_{1}, \mathcal{B}_{0}}=i_{m_{1}}\left(\mathcal{B}_{0}, \mathcal{T}_{m_{1}} \mathcal{B}_{0}\right)+\min \left(i_{m_{1}}\left(\mathcal{B}_{0}, \mathcal{T}_{m_{1}} \mathcal{B}_{0}\right)-2 e_{\mathcal{B}_{0}}, 0\right)$ if $\mathcal{T}_{m_{1}} \mathcal{B}_{0}=(I S)$, $J \notin \mathcal{T}_{m_{1}} \mathcal{B}_{0}$ and $m_{1} \notin\{I, S\}$.

$h_{m_{1}, \mathcal{B}_{0}}=i_{m_{1}}\left(\mathcal{B}_{0}, \mathcal{T}_{m_{1}} \mathcal{B}_{0}\right)+\min \left(i_{m_{1}}\left(\mathcal{B}_{0}, \mathcal{T}_{m_{1}} \mathcal{B}_{0}\right)-2 e_{\mathcal{B}_{0}}, 0\right)$ if $\mathcal{T}_{m_{1}} \mathcal{B}_{0}=(J S)$, $I \notin \mathcal{T}_{m_{1}} \mathcal{B}_{0}$ and $m_{1} \notin\{J, S\}$.

(5) $h_{m_{1}, \mathcal{B}_{0}}=i_{m_{1}}\left(\mathcal{B}_{0}, \mathcal{T}_{m_{1}} \mathcal{B}_{0}\right)$ if $\mathcal{T}_{m_{1}} \mathcal{B}_{0}=(I S), J \notin \mathcal{T}_{m_{1}} \mathcal{B}_{0}$ and $m_{1} \in\{I, S\}$.

$h_{m_{1}, \mathcal{B}_{0}}=i_{m_{1}}\left(\mathcal{B}_{0}, \mathcal{T}_{m_{1}} \mathcal{B}_{0}\right)$ if $\mathcal{T}_{m_{1}} \mathcal{B}_{0}=(J S), I \notin \mathcal{T}_{m_{1}} \mathcal{B}_{0}$ and $m_{1} \in\{J, S\}$.

(6) $h_{m_{1}, \mathcal{B}_{0}}=i_{m_{1}}\left(\mathcal{B}_{0}, \mathcal{T}_{m_{1}} \mathcal{B}_{0}\right)-e_{\mathcal{B}_{0}}$ if $\mathcal{T}_{m_{1}} \mathcal{B}_{0}=(I J)$, that $S \notin \mathcal{T}_{m_{1}} \mathcal{B}_{0}$ and $m_{1} \notin$ $\{I, J\}$.

(7) $h_{m_{1}, \mathcal{B}_{0}}=i_{m_{1}}\left(\mathcal{B}_{0}, \mathcal{T}_{m_{1}} \mathcal{B}_{0}\right)$ if $\mathcal{T}_{m_{1}} \mathcal{B}_{0}=(I J)$, that $S \notin \mathcal{T}_{m_{1}} \mathcal{B}_{0}$ and $m_{1} \in\{I, J\}$.

(8) $h_{m_{1}, \mathcal{B}_{0}}=2 i_{m_{1}}\left(\mathcal{B}_{0}, \mathcal{T}_{m_{1}} \mathcal{B}_{0}\right)-2 e_{\mathcal{B}_{0}}$ if $I, J, S \in \mathcal{T}_{m_{1}} \mathcal{B}_{0}$ and $m_{1} \notin\{I, J, S\}$.

(9) $h_{m_{1}, \mathcal{B}_{0}}=2 i_{m_{1}}\left(\mathcal{B}_{0}, \mathcal{T}_{m_{1}} \mathcal{B}_{0}\right)-e_{\mathcal{B}_{0}}$ if $I, J, S \in \mathcal{T}_{m_{1}} \mathcal{B}_{0}$ and $m_{1} \in\{I, J\}$.

(10) $h_{m_{1}, \mathcal{B}_{0}}=2 i_{m_{1}}\left(\mathcal{B}_{0}, \mathcal{T}_{m_{1}} \mathcal{B}_{0}\right)-e_{\mathcal{B}_{0}}$ if $I, J, S \in \mathcal{T}_{m_{1}} \mathcal{B}_{0}, m_{1}=$ Sand $_{m_{1}}\left(\mathcal{B}_{0}, \mathcal{T}_{m_{1}} \mathcal{B}_{0}\right) \neq$ $2 e_{\mathcal{B}_{0}}$.

(11) $h_{m_{1}, \mathcal{B}_{0}}=e_{\mathcal{B}_{0}}\left(1+\min \left(\beta_{1}, 3\right)\right)$ if $I, J, S \in \mathcal{T}_{m_{1}} \mathcal{B}_{0}, m_{1}=S$ and $i_{m_{1}}\left(\mathcal{B}_{0}, \mathcal{T}_{m_{1}} \mathcal{B}_{0}\right)=$ $2 e_{\mathcal{B}_{0}}, e_{\mathcal{B}_{0}} \beta_{1}=i_{m_{1}}\left(\mathcal{B}_{0}, O s c_{m_{1}}\left(\mathcal{B}_{0}\right)\right)$, where Osc $c_{m_{1}}\left(\mathcal{B}_{0}\right)$ is any osculating smooth algebraic curve to $\mathcal{B}_{0}$ at $m_{1}$ (the last formula of $h_{m_{1}, \mathcal{B}_{0}}$ holds if we replace $e_{\mathcal{B}_{0}} \beta_{1}$ by the first characteristic exponent of $\mathcal{B}_{0}$ non multiple of $e_{\mathcal{B}_{0}}$, see [25]).

Proof. We take $M$ such that $\mathcal{T}_{\mathcal{B}}=V(y)$ (with $\mathcal{B}=M^{-1}\left(\mathcal{B}_{0}\right)$ ). To simplify notations, we omit indices $\mathcal{B}$ in $W_{\mathbf{P}, i, \mathcal{B}}$ and consider $i \in\left\{1, \ldots, e_{\mathcal{B}}\right\}$.

- Suppose that $I, J, S \notin \mathcal{T}_{m_{1}} \mathcal{B}_{0}$. Then $W_{\mathbf{B}, i}(0)=y_{B} \neq 0, W_{\mathbf{A}, i}(0)=y_{A} \neq 0$ and $W_{\mathbf{S}^{\prime}, i}(0)=y_{S^{\prime}} \neq 0$ so

$$
\begin{aligned}
\hat{R}_{i}(0) & =\left(\begin{array}{l}
0 \\
0 \\
1
\end{array}\right) \wedge\left[y_{A} y_{B} \cdot \mathbf{S}^{\prime}-y_{A} y_{S^{\prime}} \cdot \mathbf{B}-y_{B} y_{S^{\prime}} \cdot \mathbf{A}\right] \\
& =\left(\begin{array}{c}
y_{A} y_{B} x_{S^{\prime}} y_{S^{\prime}}-y_{A} y_{S^{\prime}} x_{B}-y_{B} y_{S^{\prime}} x_{A} \\
0
\end{array}\right) .
\end{aligned}
$$

Hence $h_{m_{1}, i, \mathcal{B}}=0$ and the sum over $i=1, \ldots, e_{\mathcal{B}}$ of these quantities is equal to 0 .

- Suppose $I \in \mathcal{T}_{m_{1}} \mathcal{B}_{0}, J, \mathcal{S} \notin \mathcal{T}_{m_{1}} \mathcal{B}_{0}$ and $m_{1} \neq I$. Take $M$ such that $\mathbf{S}^{\prime}(0,1,0)$, $\mathbf{A}(1,0,0), y_{B} \neq 0$. We have $W_{\mathbf{B}, i}(0)=y_{B}, W_{\mathbf{A}, i}(0)=0$ and $W_{\mathbf{S}^{\prime}, i}(0)=1$ and so $\hat{R}_{i}(0)=\left(\begin{array}{l}0 \\ 0 \\ 1\end{array}\right) \wedge\left(\begin{array}{c}-y_{B} \\ 0 \\ 0\end{array}\right)=\left(\begin{array}{c}0 \\ -y_{B} \\ 0\end{array}\right)$. Hence $h_{m_{1}, i, \mathcal{B}}=0$ and the sum over $i=1, \ldots, e_{\mathcal{B}}$ of these quantities is equal to 0 .

- Suppose $I \in \mathcal{T}_{m_{1}} \mathcal{B}_{0}, J, S \notin \mathcal{T}_{m_{1}} \mathcal{B}_{0}$ and $m_{1}=I$. Take $M$ such that $\mathbf{S}^{\prime}(0,1,0)$, $\mathbf{A}(0,0,1), y_{B} \neq 0$. We have $W_{\mathbf{B}, i}(x)=y_{B}-g_{i}^{\prime}(x) x_{B}+z_{B}\left(x g_{i}^{\prime}(x)-g_{i}(x)\right)$, 


$$
\begin{aligned}
W_{\mathbf{A}, i}(x) & =x g_{i}^{\prime}(x)-g_{i}(x) \text { and } W_{\mathbf{S}^{\prime}, i}(x)=1 \text { and so } \\
\hat{R}_{i}(x)= & \left(\begin{array}{c}
x \\
g_{i}(x) \\
1
\end{array}\right) \wedge\left(\begin{array}{c}
-\left(x g_{i}^{\prime}(x)-g_{i}\right) x_{B} \\
\left(x g_{i}^{\prime}(x)-g_{i}\right)\left(-g_{i}^{\prime}(x) x_{B}+z_{B}\left(x g_{i}^{\prime}(x)-g_{i}(x)\right)\right) \\
-y_{B}+g_{i}^{\prime}(x) x_{B}-2 z_{B}\left(x g_{i}^{\prime}(x)-g_{i}(x)\right)
\end{array}\right) \\
= & \left(\begin{array}{c}
-y_{B} g_{i}(x)+x\left(g_{i}^{\prime}(x)\right)^{2} x_{B}-z_{B}\left(\left(x g_{i}^{\prime}(x)\right)^{2}-\left(g_{i}(x)\right)^{2}\right) \\
-x_{B}\left(2 x g_{i}^{\prime}(x)-g_{i}(x)\right)+x y_{B}+2 x z_{B}\left(x g_{i}^{\prime}(x)-g_{i}(x)\right) \\
-x_{B}\left(x g_{i}^{\prime}(x)-g_{i}(x)\right)^{2}+z_{B}\left(x g_{i}^{\prime}(x)-g_{i}(x)\right)
\end{array}\right),
\end{aligned}
$$

the valuation of the coordinates of which are larger than or equal to 1 and the valuation of the second coordinate is 1 . Hence $h_{m_{1}, i, \mathcal{B}}=1$ and the sum over $i=1, \ldots, e_{\mathcal{B}}=e_{\mathcal{B}_{0}}$ of these quantities is equal to $e_{\mathcal{B}_{0}}$.

- Suppose $S \in \mathcal{T}_{m_{1}} \mathcal{B}_{0}, I, J \notin \mathcal{T}_{m_{1}} \mathcal{B}_{0}$ and $m_{1} \neq S$. Take $M$ such that $\mathbf{A}(0,1,0)$, $\mathbf{S}^{\prime}(1,0,0), y_{B} \neq 0$. We have $W_{\mathbf{B}, i}(0)=y_{B} \neq 0, W_{\mathbf{S}^{\prime}, i}(0)=0$ and $W_{\mathbf{A}, i}(0)=1$ and so $\hat{R}_{i}(0)=\left(\begin{array}{l}0 \\ 0 \\ 1\end{array}\right) \wedge\left(\begin{array}{c}y_{B} \\ 0 \\ 0\end{array}\right)=\left(\begin{array}{c}0 \\ y_{B} \\ 0\end{array}\right)$. Hence $h_{m_{1}, i, \mathcal{B}}=0$ and the sum over $i=1, \ldots, e_{\mathcal{B}}$ of these quantities is equal to 0 .

- Suppose $m_{1}=S$ and $I, J \notin \mathcal{T}_{m_{1}} \mathcal{B}_{0}$. Take $M$ such that $\mathbf{S}^{\prime}(0,0,1), \mathbf{A}(0,1,0)$, $y_{B} \neq 0$. We have $W_{\mathbf{B}, i}(x)=y_{B}-g_{i}^{\prime}(x) x_{B}+z_{B}\left(x g_{i}^{\prime}(x)-g_{i}(x)\right), W_{\mathbf{S}^{\prime}, i}(x)=$ $x g_{i}^{\prime}(x)-g_{i}(x)$ and $W_{\mathbf{A}, i}(x)=1$ and so

$$
\begin{aligned}
& \hat{R}_{i}(x)=\left(\begin{array}{c}
x \\
g_{i}(x) \\
1
\end{array}\right) \wedge\left(\begin{array}{c}
-\left(x g_{i}^{\prime}(x)-g_{i}\right) x_{B} \\
\left.-\left(x g_{i}^{\prime}(x)-g_{i}(x)\right)\left(2 y_{B}-g_{i}^{\prime}(x) x_{B}+z_{B}\left(x g_{i}^{\prime}(x)-g_{i}(x)\right)\right)\right) \\
y_{B}-g_{i}^{\prime}(x) x_{B}
\end{array}\right) \\
& =\left(\begin{array}{c}
g_{i}(x)\left(y_{B}-g_{i}^{\prime}(x) x_{B}\right)+\left(x g_{i}^{\prime}(x)-g_{i}(x)\right)\left(2 y_{B}-g_{i}^{\prime}(x) x_{B}+z_{B}\left(x g_{i}^{\prime}(x)-g_{i}(x)\right)\right) \\
-\left(x g_{i}^{\prime}(x)-g_{i}(x)\right) x_{B}-x\left(y_{B}-g_{i}^{\prime}(x) x_{B}\right) \\
-\left(x g_{i}^{\prime}(x)-g_{i}(x)\right)\left(2 x y_{B}-g_{i}^{\prime}(x) x x_{B}+z_{B} x\left(x g_{i}^{\prime}(x)-g_{i}(x)\right)+g_{i}(x)\left(x g_{i}^{\prime}(x)-g_{i}(x)\right) x_{B}\right)
\end{array}\right),
\end{aligned}
$$

the valuation of the coordinates of which are larger than or equal to 1 and the valuation of the second coordinate is 1 . Hence $h_{m_{1}, i, \mathcal{B}}=1$ and the sum over $i=1, \ldots, e_{\mathcal{B}}$ of these quantities is equal to $e_{\mathcal{B}_{0}}$.

- Suppose $\mathcal{T}_{m_{1}} \mathcal{B}_{0}=(I S), J \notin \mathcal{T}_{m_{1}} \mathcal{B}_{0}$ and $m_{1} \notin\{I, S\}$. Take $M$ such that $\mathbf{S}^{\prime}(1,0,0), \mathbf{B}(0,1,0), y_{A}=0, x_{A} \neq 0, z_{A} \neq 0$. We have $W_{\mathbf{S}^{\prime}, i}(x)=-g_{i}^{\prime}(x)$, $W_{\mathbf{A}, i}(x)=-g_{i}^{\prime}(x) x_{A}+z_{A}\left(x g_{i}^{\prime}(x)-g_{i}(x)\right)$ and $W_{\mathbf{B}, i}(x)=1$ and so

$$
\begin{aligned}
\hat{R}_{i}(x)= & \left(\begin{array}{c}
x \\
g_{i}(x) \\
1
\end{array}\right) \wedge\left(\begin{array}{c}
z_{A}\left(x g_{i}^{\prime}(x)-g_{i}(x)\right) \\
-\left(g_{i}^{\prime}(x)\right)^{2} x_{A}+g_{i}^{\prime}(x)\left(x g_{i}^{\prime}(x)-g_{i}(x)\right) z_{A} \\
g_{i}^{\prime}(x) z_{A}
\end{array}\right) \\
= & \left(\begin{array}{c}
g_{i}(x) g_{i}^{\prime}(x) z_{A}+\left(g_{i}^{\prime}(x)\right)^{2} x_{A}-g_{i}^{\prime}(x)\left(x g_{i}^{\prime}(x)-g_{i}(x)\right) z_{A} \\
-g_{i}(x) z_{A} \\
-x\left(g_{i}^{\prime}(x)\right)^{2} x_{A}+\left(x g_{i}^{\prime}(x)-g_{i}(x)\right)^{2} z_{A}
\end{array}\right),
\end{aligned}
$$

the valuation of the coordinates of which are respectively $2 \operatorname{val}\left(g_{i}\right)-2, \operatorname{val}\left(g_{i}\right)$ and $2 \operatorname{val}\left(g_{i}\right)-1$. Hence $h_{m_{1}, i, \mathcal{B}}=\operatorname{val}\left(g_{i}\right)+\min \left(\operatorname{val}\left(g_{i}\right)-2,0\right)$ and the 
sum over $i=1, \ldots, e_{\mathcal{B}}$ of these quantities is equal to $i_{m_{1}}\left(\mathcal{B}_{0}, \mathcal{T}_{m_{1}} \mathcal{B}_{0}\right)+$ $\min \left(i_{m_{1}}\left(\mathcal{B}_{0}, \mathcal{T}_{m_{1}} \mathcal{B}_{0}\right)-2 e_{\mathcal{B}_{0}}, 0\right)$.

- Suppose $\mathcal{T}_{m_{1}} \mathcal{B}_{0}=(I S), J \notin \mathcal{T}_{m_{1}} \mathcal{B}_{0}$ and $m_{1}=I$. Take $M$ such that $\mathbf{S}^{\prime}(1,0,0)$, $\mathbf{B}(0,1,0), \mathbf{A}(0,0,1)$. We have $W_{\mathbf{S}^{\prime}, i}(x)=-g_{i}^{\prime}(x), W_{\mathbf{A}, i}(x)=x g_{i}^{\prime}(x)-g_{i}(x)$ and $W_{\mathbf{B}, i}(x)=1$ and so

$$
\hat{R}_{i}(x)=\left(\begin{array}{c}
x \\
g_{i}(x) \\
1
\end{array}\right) \wedge\left(\begin{array}{c}
x g_{i}^{\prime}(x)-g_{i}(x) \\
g_{i}^{\prime}(x)\left(x g_{i}^{\prime}(x)-g_{i}(x)\right) \\
g_{i}^{\prime}(x)
\end{array}\right)=\left(\begin{array}{c}
g_{i}^{\prime}(x)\left(2 g_{i}(x)-x g_{i}^{\prime}(x)\right) \\
-g_{i}(x) \\
\left(x g_{i}^{\prime}(x)-g_{i}(x)\right)^{2}
\end{array}\right),
$$

the valuation of the coordinates of which are larger than or equal to $\operatorname{val}\left(g_{i}\right)$, the second coordinate has valuation $\operatorname{val}\left(g_{i}\right)$. Hence $h_{m_{1}, i, \mathcal{B}}=\operatorname{val}\left(g_{i}\right)$ and the sum over $i=1, \ldots, e_{\mathcal{B}}$ of these quantities is equal to $i_{m_{1}}\left(\mathcal{B}_{0}, \mathcal{T}_{m_{1}} \mathcal{B}_{0}\right)$.

- Suppose $\mathcal{T}_{m_{1}} \mathcal{B}_{0}=(I S), J \notin \mathcal{T}_{m_{1}} \mathcal{B}_{0}$ and $m_{1}=S$. Take $M$ such that $\mathbf{A}(1,0,0)$, $\mathbf{B}(0,1,0), \mathbf{S}^{\prime}(0,0,1)$. We have $W_{\mathbf{S}^{\prime}, i}(x)=x g_{i}^{\prime}(x)-g_{i}(x), W_{\mathbf{A}, i}(x)=-g_{i}^{\prime}(x)$ and $W_{\mathbf{B}, i}(x)=1$ and so

$$
\hat{R}_{i}(x)=\left(\begin{array}{c}
x \\
g_{i}(x) \\
1
\end{array}\right) \wedge\left(\begin{array}{c}
-\left(x g_{i}^{\prime}(x)-g_{i}(x)\right) \\
g_{i}^{\prime}\left(x g_{i}^{\prime}(x)-g_{i}(x)\right) \\
-g_{i}^{\prime}(x)
\end{array}\right)=\left(\begin{array}{c}
-x\left(g_{i}^{\prime}(x)\right)^{2} \\
g_{i}(x) \\
\left(x g_{i}^{\prime}(x)\right)^{2}-\left(g_{i}(x)\right)^{2}
\end{array}\right)
$$

the valuation of the coordinates of which being larger than or equal to $\operatorname{val}\left(g_{i}\right)$ and the valuation of the second coordinate is equal to $\operatorname{val}\left(g_{i}\right)$. Hence $h_{m_{1}, i, \mathcal{B}}=\operatorname{val}\left(g_{i}\right)$ and the sum over $i=1, \ldots, e_{\mathcal{B}}$ of these quantities is equal to $i_{m_{1}}\left(\mathcal{B}_{0}, \mathcal{T}_{m_{1}} \mathcal{B}_{0}\right)$.

- Suppose $\mathcal{T}_{m_{1}} \mathcal{B}_{0}=(I J), S \notin \mathcal{T}_{m_{1}} \mathcal{B}_{0}$ and $m_{1} \notin\{I, J\}$. Take $M$ such that $\mathbf{S}^{\prime}(0,1,0), \mathbf{B}(1,0,0), y_{A}=0, x_{A} \neq 0, z_{A} \neq 0$. We have $W_{\mathbf{B}, i}(x)=-g_{i}^{\prime}(x)$, $W_{\mathbf{A}, i}(x)=-g_{i}^{\prime}(x) x_{A}+z_{A}\left(x g_{i}^{\prime}(x)-g_{i}(x)\right)$ and $W_{\mathbf{S}^{\prime}, i}(x)=1$ and so

$$
\begin{aligned}
\hat{R}_{i}(x) & =\left(\begin{array}{c}
x \\
g_{i}(x) \\
1
\end{array}\right) \wedge\left(\begin{array}{c}
2 g_{i}^{\prime}(x) x_{A}-z_{A}\left(x g_{i}^{\prime}(x)-g_{i}(x)\right) \\
\left(g_{i}^{\prime}(x)\right)^{2} x_{A}-g_{i}^{\prime}(x)\left(x g_{i}^{\prime}(x)-g_{i}(x)\right) z_{A} \\
g_{i}^{\prime}(x) z_{A}
\end{array}\right) \\
& =\left(\begin{array}{c}
\left(g_{i}^{\prime}(x)\right)^{2}\left(x z_{A}-x_{A}\right) \\
-z_{A}\left(x g_{i}^{\prime}(x)-g_{i}(x)\right)^{2}+x_{A} g_{i}^{\prime}(x)\left(x g_{i}^{\prime}(x)-2 g_{i}(x)\right)
\end{array}\right),
\end{aligned}
$$

the valuation of the coordinates of which are respectively $2 \operatorname{val}\left(g_{i}\right)-2, \operatorname{val}\left(g_{i}\right)-1$ and larger than $\operatorname{val}\left(g_{i}\right)$. Hence $h_{m_{1}, i, \mathcal{B}}=\operatorname{val}\left(g_{i}\right)-1$ and the sum over $i=$ $1, \ldots, e_{\mathcal{B}}$ of these quantities is equal to $i_{m_{1}}\left(\mathcal{B}_{0}, \mathcal{T}_{m_{1}} \mathcal{B}_{0}\right)-e_{\mathcal{B}_{0}}$.

- Suppose that $\mathcal{T}_{m_{1}} \mathcal{B}_{0}=(I J)$, that $S \notin \mathcal{T}_{m_{1}} \mathcal{B}_{0}$ and $m_{1}=I$. Take $M$ such that $\mathbf{S}^{\prime}(0,1,0), \mathbf{B}(1,0,0), \mathbf{A}(0,0,1)$. We have $W_{\mathbf{B}, i}(x)=-g_{i}^{\prime}(x), W_{\mathbf{A}, i}(x)=$ $x g_{i}^{\prime}(x)-g_{i}(x)$ and $W_{\mathbf{S}^{\prime}, i}(x)=1$ and so

$$
\hat{R}_{i}(x)=\left(\begin{array}{c}
x \\
g_{i}(x) \\
1
\end{array}\right) \wedge\left(\begin{array}{c}
-\left(x g_{i}^{\prime}(x)-g_{i}(x)\right) \\
-g_{i}^{\prime}(x)\left(x g_{i}^{\prime}(x)-g_{i}(x)\right) \\
g_{i}^{\prime}(x)
\end{array}\right)=\left(\begin{array}{c}
x\left(g_{i}^{\prime}(x)\right)^{2} \\
-\left(2 x g_{i}^{\prime}(x)-g_{i}(x)\right) \\
-\left(x g_{i}^{\prime}(x)-g_{i}(x)\right)^{2}
\end{array}\right)
$$


the valuation of the coordinates of which being larger than or equal to $\operatorname{val}\left(g_{i}\right)$ and the valuation of the second coordinate is equal to $\operatorname{val}\left(g_{i}\right)$. Hence $h_{m_{1}, i, \mathcal{B}}=\operatorname{val}\left(g_{i}\right)$ and the sum over $i=1, \ldots, e_{\mathcal{B}}$ of these quantities is equal to $i_{m_{1}}\left(\mathcal{B}_{0}, \mathcal{T}_{m_{1}} \mathcal{B}_{0}\right)$.

- Suppose that $I, J, S \in \mathcal{T}_{m_{1}} \mathcal{B}_{0}$ and $m_{1} \notin\{I, J, S\}$. Take $M$ such that $\mathbf{S}^{\prime}(1,0,0)$, $y_{A}=y_{B}=0, x_{A} \neq 0, z_{A} \neq 0, x_{B} \neq 0, z_{B} \neq 0, x_{A} z_{B} \neq x_{B} z_{A}$. We have $W_{\mathbf{S}^{\prime}, i}(x)=-g_{i}^{\prime}(x), W_{\mathbf{A}, i}(x)=-g_{i}^{\prime}(x) x_{A}+z_{A}\left(x g_{i}^{\prime}(x)-g_{i}(x)\right)$ and $W_{\mathbf{B}, i}(x)=$ $-g_{i}^{\prime}(x) x_{B}+z_{B}\left(x g_{i}^{\prime}(x)-g_{i}(x)\right)$ and so

$$
\begin{aligned}
\hat{R}_{i}(x)= & \left(\begin{array}{c}
x \\
g_{i}(x) \\
1
\end{array}\right) \wedge\left(\begin{array}{c}
-x_{A}\left(g_{i}^{\prime}(x)\right)^{2} x_{B}+z_{A} z_{B}\left(x g_{i}^{\prime}(x)-g_{i}(x)\right)^{2} \\
0 \\
-\left(g_{i}^{\prime}(x)\right)^{2}\left(x z_{B}+x_{B} z_{A}\right)_{A}+2 z_{A} z_{B} g_{i}^{\prime}(x)\left(x g_{i}^{\prime}(x)-g_{i}(x)\right)
\end{array}\right) \\
= & \left(\begin{array}{c}
-g_{i}(x)\left(g_{i}^{\prime}(x)\right)^{2}\left(x z_{B}+x_{B} z_{A}\right)_{A}+2 z_{A} z_{B} g_{i}(x) g_{i}^{\prime}(x)\left(x g_{i}^{\prime}(x)-g_{i}(x)\right) \\
-x_{A}\left(g_{i}^{\prime}(x)\right)^{2} x_{B}+z_{A} z_{B}\left(x g_{i}^{\prime}(x)-g_{i}(x)\right)^{2}-x[\ldots] \\
x_{A} g_{i}(x)\left(g_{i}^{\prime}(x)\right)^{2} x_{B}-z_{A} z_{B} g_{i}(x)\left(x g_{i}^{\prime}(x)-g_{i}(x)\right)^{2}
\end{array}\right),
\end{aligned}
$$

the valuation of the coordinates of which are larger than or equal to $2 \operatorname{val}\left(g_{i}\right)-$ 2 , the valuation of the second coodinate is $2 \mathrm{val}\left(g_{i}\right)-2$. Hence $h_{m_{1}, i, \mathcal{B}}=$ $2 \operatorname{val}\left(g_{i}\right)-2$ and the sum over $i=1, \ldots, e_{\mathcal{B}}$ of these quantities is equal to $2 i_{m_{1}}\left(\mathcal{B}_{0}, \mathcal{T}_{m_{1}} \mathcal{B}_{0}\right)-2 e_{\mathcal{B}_{0}}$.

- Suppose that $I, J, S \in \mathcal{T}_{m_{1}} \mathcal{B}_{0}$ and $m_{1}=J$. Take $M$ such that $\mathbf{B}(0,0,1)$, $\mathbf{S}^{\prime}(1,0,0), y_{A}=0, x_{A} \neq 0$ and $z_{A} \neq 0$. We have $W_{\mathbf{S}^{\prime}, i}(x)=-g_{i}^{\prime}(x)$, $W_{\mathbf{A}, i}(x)=-g_{i}^{\prime}(x) x_{A}+z_{A}\left(x g_{i}^{\prime}(x)-g_{i}(x)\right)$ and $W_{\mathbf{B}, i}(x)=x g_{i}^{\prime}(x)-g_{i}(x)$ and so

$$
\begin{aligned}
\hat{R}_{i}(x) & =\left(\begin{array}{c}
x \\
g_{i}(x) \\
1
\end{array}\right) \wedge\left(\begin{array}{c}
z_{A}\left(x g_{i}^{\prime}(x)-g_{i}(x)\right)^{2} \\
0 \\
-x_{A}\left(g_{i}^{\prime}(x)\right)^{2}+2 z_{A}\left(x g_{i}^{\prime}(x)-g_{i}(x)\right) g_{i}^{\prime}(x)
\end{array}\right) \\
& =\left(\begin{array}{c}
g_{i}(x) g_{i}^{\prime}(x)\left(-g_{i}^{\prime}(x) x_{A}+2 z_{A}\left(x g_{i}^{\prime}(x)-g_{i}(x)\right)\right) \\
\left.z_{A}\left(x g_{i}^{\prime}(x)-g_{i}(x)\right)^{2}-x g_{i}^{\prime}(x)\left(-g_{i}^{\prime}(x) x_{A}+2 z_{A}\left(x g_{i}^{\prime}(x)-g_{i}(x)\right)\right)\right), \\
-g_{i}(x) z_{A}\left(x g_{i}^{\prime}(x)-g_{i}(x)\right)^{2}
\end{array}\right),
\end{aligned}
$$

the valuation of the coordinates of which are larger than or equal to $2 \mathrm{val}\left(g_{i}\right)-1$ and the valuation of the second coordinate is $2 \operatorname{val}\left(g_{i}\right)-1$. Hence $h_{m_{1}, i, \mathcal{B}}=$ $2 \operatorname{val}\left(g_{i}\right)-1$ and the sum over $i=1, \ldots, e_{\mathcal{B}}$ of these quantities is equal to $2 i_{m_{1}}\left(\mathcal{B}_{0}, \mathcal{T}_{m_{1}} \mathcal{B}_{0}\right)-e_{\mathcal{B}_{0}}$.

- Suppose that $I, J, S \in \mathcal{T}_{m_{1}} \mathcal{B}_{0}$ and $m_{1}=S$. Take $M$ such that $\mathbf{S}^{\prime}(0,0,1)$, $\mathbf{B}(1,0,0), y_{A}=0, x_{A} \neq 0$ and $z_{A} \neq 0$. We have $W_{\mathbf{B}, i}(x)=-g_{i}^{\prime}(x)$, $W_{\mathbf{A}, i}(x)=-g_{i}^{\prime}(x) x_{A}+z_{A}\left(x g_{i}^{\prime}(x)-g_{i}(x)\right)$ and $W_{\mathbf{S}^{\prime}, i}(x)=x g_{i}^{\prime}(x)-g_{i}(x)$ and so

$$
\begin{aligned}
\hat{R}_{i}(x) & =\left(\begin{array}{c}
x \\
g_{i}(x) \\
1
\end{array}\right) \wedge\left(\begin{array}{c}
2 x_{A} g_{i}^{\prime}(x)\left(x g_{i}^{\prime}(x)-g_{i}(x)\right)-z_{A}\left(x g_{i}^{\prime}(x)-g_{i}(x)\right)^{2} \\
0 \\
\left(g_{i}^{\prime}(x)\right)^{2} x_{A}
\end{array}\right) \\
& =\left(\begin{array}{c}
x_{A} g_{i}^{\prime}(x)\left(x g_{i}^{\prime}(x)-2 g_{i}(x)\right)-z_{A}\left(x g_{i}^{\prime}(x)-g_{i}(x)\right)^{2} \\
g_{i}(x)\left(g_{i}^{\prime}(x)\right)^{2} x_{A} \\
-2 x_{A} g_{i}(x) g_{i}^{\prime}(x)\left(x g_{i}^{\prime}(x)-g_{i}(x)\right)+z_{A} g_{i}(x)\left(x g_{i}^{\prime}(x)-g_{i}(x)\right)^{2}
\end{array}\right) .
\end{aligned}
$$


The valuation of the first coordinate is $3 \operatorname{val}\left(g_{i}\right)-2$ is smaller than or equal to the valuation of the third coordinate.

If $\operatorname{val}\left(g_{i}\right) \neq 2$, the valuation of the second coordinate is $2 \operatorname{val}\left(g_{i}\right)-1$; hence $h_{m_{1}, i, \mathcal{B}}=2 \operatorname{val}\left(g_{i}\right)-1$ and the sum over $i=1, \ldots, e_{\mathcal{B}}$ of these quantities is equal to $2 i_{m_{1}}\left(\mathcal{B}_{0}, \mathcal{T}_{m_{1}} \mathcal{B}_{0}\right)-e_{\mathcal{B}_{0}}$.

Suppose now that $\operatorname{val}\left(g_{i}\right)=2$, then $3 \operatorname{val}\left(g_{i}\right)-2=4$ and there exist $\alpha, \alpha_{1} \in \mathbb{C}$ and $\beta_{1}>2$ such that $g_{i}(x)=\alpha x^{2}+\alpha_{1} x^{\beta_{1}}+\ldots$ Then, the second coordinate has the following form $\left(x_{A} 2 \alpha\left(\beta_{1}-2\right) x^{\beta_{1}+1}+\ldots\right)+x^{4}(\ldots)$. Therefore $h_{m_{1}, i, \mathcal{B}}=$ $\min \left(\beta_{1}+1,4\right)$ and the sum over $i=1, \ldots, e_{\mathcal{B}}$ of these quantities is equal to $e_{\mathcal{B}_{0}}\left(1+\min \left(\beta_{1}, 3\right)\right)$.

Proof of Theorem 3.1. Recall that (6.4) says

$$
\operatorname{mclass}\left(\Sigma_{S}(\mathcal{C})\right)=2 d^{\vee}+d-\sum_{m_{1} \in \mathcal{C}} \sum_{\mathcal{B}_{0} \in \operatorname{Branch}_{m_{1}}(\mathcal{C})} h_{m_{1}, \mathcal{B}_{0}}
$$

and that the values of $h_{m_{1}, \mathcal{B}_{0}}$ have been given in Lemma 6.5.

- Assume first $S \notin \ell_{\infty}$. Then we have to sum the $h_{m_{1}, \mathcal{B}_{0}}$ coming from Items 3,4 , 5, 6 and 7 of Lemma 6.5 .

The sum of the $h_{m_{1}, \mathcal{B}_{0}}$ coming from Items 3 and 5 applied with $m_{1}=S$ gives directly $g^{\prime}$.

The sum of the $h_{m_{1}, \mathcal{B}_{0}}$ coming from Items 3, 5 and 7 applied with $m_{1} \in\{I, J\}$ gives $f+\Omega_{I}\left(\mathcal{C}, \ell_{\infty}\right)+\Omega_{J}\left(\mathcal{C}, \ell_{\infty}\right)$.

The sum of the $h_{m_{1}, \mathcal{B}_{0}}$ coming from Item 6 gives $g-\Omega_{I}\left(\mathcal{C}, \ell_{\infty}\right)-\Omega_{J}\left(\mathcal{C}, \ell_{\infty}\right)$. The sum of the $h_{m_{1}, \mathcal{B}_{0}}$ coming from Item 4 gives $2 f^{\prime}-q^{\prime}$ (notice that $h_{m_{1}, \mathcal{B}_{0}}=$ $\left.2\left(i_{m_{1}}\left(\mathcal{B}_{0}, \mathcal{T}_{m_{1}} \mathcal{B}_{0}\right)-e_{\mathcal{B}_{0}}\right)-\left(i_{m_{1}}\left(\mathcal{B}_{0}, \mathcal{T}_{m_{1}} \mathcal{B}_{0}\right)-2 e_{\mathcal{B}_{0}}\right) \mathbf{1}_{i_{m_{1}}}\left(\mathcal{B}_{0}, \mathcal{T}_{m_{1}} \mathcal{B}_{0}\right) \geq 2 e_{\mathcal{B}_{0}}\right)$.

- Assume first $S \notin \ell_{\infty}$. Then we have to sum the $h_{m_{1}}, \mathcal{B}_{0}$ coming from Items 3,8 , 9, 10 and 11 of Lemma 6.5.

The sum of the $h_{m_{1}, \mathcal{B}_{0}}$ coming from Items 3 (with $m_{1}=S$ ), 10 and 11 gives $2 \Omega_{S}\left(\mathcal{C}, \ell_{\infty}\right)+\mu_{S}+c^{\prime}(S)$.

The sum of the $h_{m_{1}, \mathcal{B}_{0}}$ coming from Items 3 and 9 applied with $m_{1} \in\{I, J\}$ gives $2\left(\Omega_{I}\left(\mathcal{C}, \ell_{\infty}\right)+\Omega_{J}\left(\mathcal{C}, \ell_{\infty}\right)\right)+\mu_{I}+\mu_{J}$.

The sum of the $h_{m_{1}, \mathcal{B}_{0}}$ coming from Item 8 gives $2\left(g-\Omega_{I}\left(\mathcal{C}, \ell_{\infty}\right)-\Omega_{J}\left(\mathcal{C}, \ell_{\infty}\right)-\right.$ $\left.\Omega_{S}\left(\mathcal{C}, \ell_{\infty}\right)\right)$.

\section{Appendix}

\section{A. Intersection numbers of curves and pro-branches}

The following result expresses the classical intersection number $i_{m_{1}}\left(\mathcal{C}, \mathcal{C}^{\prime}\right)$ defined in [15, page 54] thanks to the use of probranches.

Proposition A.1. Let $m \in \mathbb{P}^{2}$. Let $\mathcal{C}=V(F)$ and $\mathcal{C}^{\prime}=V\left(F^{\prime}\right)$ be two algebraic plane curves containing $m$, with homogeneous polynomials $F, F^{\prime} \in \mathbb{C}[X, Y, Z]$. 
Let $M \in G L\left(\mathbb{C}^{3}\right)$ be such that $\Pi\left(M\left(\mathbf{P}_{0}\right)\right)=m$ and such that the tangent cones of $V(F \circ M)$ and of $V\left(F^{\prime} \circ M\right)$ do not contain $V(X)$.

Assume that $V(F \circ M)$ admits $b$ branches at $P_{0}$ and that its $\beta$-th branch $\mathcal{B}_{\beta}$ has multiplicity $e_{\beta}$. Assume that $V\left(F^{\prime} \circ M\right)$ admits $b^{\prime}$ branches at $P_{0}$ and that its $\beta^{\prime}$-th branch $\mathcal{B}_{\beta^{\prime}}^{\prime}$ has multiplicity $e_{\beta^{\prime}}^{\prime}$.

Then we have

$$
i_{m}\left(\mathcal{C}, \mathcal{C}^{\prime}\right)=\sum_{\beta=1}^{b} \sum_{j=0}^{e_{\beta}-1} \sum_{\beta^{\prime}=1}^{b^{\prime}} \sum_{j^{\prime}=0}^{e_{\beta^{\prime}}^{\prime}-1} \operatorname{val}_{x}\left[h_{\beta}\left(\zeta^{j} x^{\frac{1}{e_{\beta}}}\right)-h_{\beta^{\prime}}^{\prime}\left(\zeta^{\prime j^{\prime}} x^{\frac{1}{e_{\beta^{\prime}}^{\prime}}}\right)\right]
$$

with $y=h_{\beta}\left(\zeta^{j} x^{\frac{1}{e_{\beta}}}\right) \in \mathbb{C}\left\langle x^{*}\right\rangle$ an equation of the $j$-th probranch of $\mathcal{B}_{\beta}$ at $P_{0}$, $y=h_{\beta^{\prime}}^{\prime}\left(\zeta^{\prime j^{\prime}} x^{\frac{1}{e_{\beta^{\prime}}^{\prime}}}\right) \in \mathbb{C}\left\langle x^{*}\right\rangle$ an equation of the $k^{\prime}$-th probranch of $\mathcal{B}_{\beta^{\prime}}^{\prime}$ at $P_{0}$, with $\zeta:=e^{\frac{2 i \pi}{e_{\beta}}}$ and $\zeta^{\prime}:=e^{\frac{2 i \pi}{e^{\prime}}}$

With the notations of Proposition A.1, we get

$$
i_{m}\left(\mathcal{C}, \mathcal{C}^{\prime}\right)=\sum_{\beta=1}^{b} i_{P_{0}}\left(\mathcal{B}_{\beta}, V\left(F^{\prime}\right)\right)
$$

with the usual definition given in [24] of intersection number of a branch with a curve

$$
i_{P_{0}}\left(\mathcal{B}_{\beta}, V\left(F^{\prime} \circ M\right)\right)=\sum_{j=0}^{e_{\beta}-1} \operatorname{val}_{x}\left(F^{\prime} \circ M\left(x, h_{j, \beta}\left(\zeta^{j} x^{\frac{1}{e_{\beta}}}\right)\right)\right) .
$$

Proof of Proposition A.1. By definition, the intersection number is defined by

$$
i_{m}\left(\mathcal{C}, \mathcal{C}^{\prime}\right)=i_{P_{0}}\left(V\left(F \circ M, F^{\prime} \circ M\right)=\operatorname{length}\left(\left(\frac{\mathbb{C}[X, Y, Z]}{\left(F \circ M, F^{\prime} \circ M\right)}\right)_{(X, Y, Z)}\right)\right.
$$

where $\left(\frac{\mathbb{C}[X, Y, Z]}{\left(F \circ M, F^{\prime} \circ M\right)}\right)_{(X, Y, Z)}$ is the local ring in the maximal ideal $(X, Y, Z)$ of $P_{0}$ [15, page 53]. According to [13], we have

$$
i_{m}\left(\mathcal{C}, \mathcal{C}^{\prime}\right)=\operatorname{dim}_{\mathbb{C}}\left(\left(\frac{\mathbb{C}[X, Y, Z]}{\left(F \circ M, F^{\prime} \circ M\right)}\right)_{(X, Y, Z)}\right)
$$

Let $f, f^{\prime}$ be defined by $f(x, y)=F \circ M(x, y, 1), f^{\prime}(x, y)=F^{\prime} \circ M(x, y, 1)$. We get

$$
i_{m}\left(\mathcal{C}, \mathcal{C}^{\prime}\right)=\operatorname{dim}_{\mathbb{C}}\left(\left(\frac{\mathbb{C}[x, y]}{\left(f, f^{\prime}\right)}\right)_{(x, y)}\right)=\operatorname{dim}_{\mathbb{C}} \frac{\mathbb{C}\langle x, y\rangle}{\left(f, f^{\prime}\right)}
$$


Recall that, according to the Weierstrass preparation theorem, there exist two units $U$ and $U^{\prime}$ of $\mathbb{C}\langle x, y\rangle$ and $f_{1}, \ldots, f_{b}, f_{1}^{\prime}, \ldots, f_{b^{\prime}}^{\prime} \in \mathbb{C}\langle x\rangle[y]$ monic irreducible such that

$$
f=U \prod_{\beta=1}^{b} f_{\beta} \text { and } f^{\prime}=U^{\prime} \prod_{\beta^{\prime}=1}^{b^{\prime}} f_{\beta^{\prime}}^{\prime},
$$

$f_{\beta}=0$ being an equation of $\mathcal{B}_{\beta}$ and $f_{\beta^{\prime}}^{\prime}=0$ being an equation of $\mathcal{B}_{\beta^{\prime}}^{\prime}$. According to the Puiseux theorem, $\mathcal{B}_{\beta}$ (respectively $\mathcal{B}_{\beta^{\prime}}^{\prime}$ ) admits a parametrization

$$
\left\{\begin{array} { c } 
{ x = t ^ { e _ { \beta } } } \\
{ x = h _ { \beta } ( t ) \in \mathbb { C } \langle t \rangle }
\end{array} \left(\text { respectively }\left\{\begin{array}{c}
x=t^{e_{\beta^{\prime}}^{\prime}} \\
x=h_{\beta^{\prime}}^{\prime}(t) \in \mathbb{C}\langle t\rangle
\end{array}\right) .\right.\right.
$$

We know that, for every $\beta \in\{1, \ldots, b\}$ and every $j \in\left\{0, . ., e_{\beta}\right\}, h_{\beta}\left(\zeta^{j} x^{\frac{1}{e_{\beta}}}\right) \in$ $\mathbb{C}\left\langle x^{\frac{1}{e_{\beta}}}\right\rangle$ are the $y$-roots of $f_{\beta}$ (respectively $h_{\beta^{\prime}}\left(\zeta^{j^{\prime}} x^{\frac{1}{e^{\prime}}}\right) \in \mathbb{C}\left\langle x^{\frac{1}{e^{\prime} \beta^{\prime}}}\right\rangle$ are the $y$-roots of $f_{\beta^{\prime}}^{\prime}$. In particular, we have

$$
f_{\beta}(x, y)=\prod_{j=0}^{e_{\beta}-1}\left(y-h_{\beta}\left(\zeta^{j} x^{\frac{1}{e_{\beta}}}\right)\right) \text { and } f_{\beta^{\prime}}^{\prime}(x, y)=\prod_{j^{\prime}=0}^{e_{\beta^{\prime}}^{\prime}-1}\left(y-h_{\beta^{\prime}}^{\prime}\left(\zeta^{\prime j^{\prime}} x^{\frac{1}{e_{\beta^{\prime}}^{\prime}}}\right)\right) \text {. }
$$

Therefore we have the following sequence of $\mathbb{C}$-algebra-isomorphisms:

$$
\frac{\mathbb{C}\langle x, y\rangle}{\left(f, f^{\prime}\right)}=\frac{\mathbb{C}\langle x, y\rangle}{\left(\prod_{\beta=1}^{b} f_{\beta}(x, y), f^{\prime}(x, y)\right)} \cong \prod_{\beta=1}^{b} A_{\beta},
$$

where $A_{\beta}:=\frac{\mathbb{C}\langle x, y\rangle}{\left(f_{\beta}(x, y), f^{\prime}(x, y)\right)}$. Let $\beta \in\{1, \ldots, b\}$. We observe that we have

$$
A_{\beta}=\prod_{j=0}^{e_{\beta}-1} \frac{\mathbb{C}\langle x\rangle}{\left(f^{\prime}\left(x, h_{\beta}\left(\xi^{j} x^{\frac{1}{e} \beta}\right)\right)\right)} .
$$

On another hand, we have

$$
\begin{aligned}
D_{\beta} & :=\frac{\mathbb{C}\left\langle x^{\frac{1}{e_{\beta}}}, y\right\rangle}{\left(f_{\beta}(x, y), f^{\prime}(x, y)\right)}=\frac{\mathbb{C}\left\langle x^{\frac{1}{e_{\beta}}}, y\right\rangle}{\left(\prod_{j=0}^{e_{\beta}-1}\left(y-h_{\beta}\left(\zeta^{j} x^{\frac{1}{e_{\beta}}}\right)\right), f^{\prime}(x, y)\right)} \\
& \cong \prod_{j=0}^{e_{\beta}-1} \frac{\mathbb{C}\left\langle x^{\frac{1}{e_{\beta}}}, y\right\rangle}{\left(y-h_{\beta}\left(\zeta^{j} x^{\frac{1}{e_{\beta}}}\right), f^{\prime}(x, y)\right)} \cong \prod_{j=0}^{e_{\beta}-1} D_{\beta, j}
\end{aligned}
$$


with

$$
D_{\beta, j}:=\frac{\mathbb{C}\left\langle x^{\frac{1}{e_{\beta}}}\right\rangle}{\left(f^{\prime}\left(x, h_{\beta}\left(\zeta^{j} x^{\frac{1}{e_{\beta}}}\right)\right)\right)} .
$$

We consider now the natural extension of rings $i_{\beta}: A_{\beta, j} \hookrightarrow D_{\beta, j}$ such that

$$
\forall g \in A_{\beta}, \quad \operatorname{val}_{x^{1 / e_{\beta}}}\left(\left(i_{\beta}(g)\right)(x)\right)=e_{\beta} \operatorname{val}_{x}(g(x)) .
$$

We have

$$
D_{\beta} \cong \prod_{j=0}^{e_{\beta}-1} \frac{\mathbb{C}\left\langle x^{\frac{1}{e_{\beta}}}\right\rangle}{\left(x^{v_{\beta}}\right)}
$$

where $v_{\beta}$ is the valuation in $x^{\frac{1}{e_{\beta}}}$ of $\left(f^{\prime}\left(x, h_{\beta}\left(\zeta^{j} x^{\frac{1}{e_{\beta}}}\right)\right)\right)$, i.e.

$$
v_{\beta}:=\operatorname{val}_{t}\left(f^{\prime}\left(t^{e_{\beta}}, h_{\beta}\left(\zeta^{j} t\right)\right)\right)=e_{\beta} \operatorname{val}_{x}\left(f^{\prime}\left(x, h_{\beta}\left(\zeta^{j} x^{\frac{1}{e_{\beta}}}\right)\right)\right) .
$$

We get

$$
\begin{aligned}
i_{m}\left(\mathcal{C}, \mathcal{C}^{\prime}\right) & =\sum_{\beta=1}^{b} \operatorname{dim}_{\mathbb{C}} A_{\beta}=\sum_{\beta=1}^{b} \sum_{j=0}^{e_{\beta}-1} \frac{1}{e_{\beta}} \operatorname{val}_{t}\left(f^{\prime}\left(t^{e_{\beta}}, h_{\beta}\left(\zeta^{j} t\right)\right)\right) \\
& =\sum_{\beta=1}^{b} \sum_{j=0}^{e_{\beta}-1} \operatorname{val}_{x}\left(f^{\prime}\left(x, h_{\beta}\left(\zeta^{j} x^{\frac{1}{e_{\beta}}}\right)\right)\right) \\
& =\sum_{\beta=1}^{b} \sum_{j=0}^{e_{\beta}-1} \sum_{\beta^{\prime}=1}^{b^{\prime}} \operatorname{val}_{x}\left(f_{\beta^{\prime}}^{\prime}\left(x, h_{\beta}\left(\zeta^{j} x^{\frac{1}{e_{\beta}}}\right)\right)\right) .
\end{aligned}
$$

Observe now that

$$
\operatorname{val}_{x}\left(f_{\beta^{\prime}}^{\prime}\left(x, h_{\beta}\left(\zeta^{j} x^{\frac{1}{e_{\beta}}}\right)\right)\right) \in \frac{1}{e_{\beta}} \mathbb{N}
$$

and that

$$
f_{\beta^{\prime}}^{\prime}\left(x, h_{\beta}\left(\zeta^{j} x^{\frac{1}{e_{\beta}}}\right)\right) \equiv \operatorname{Res}\left(f_{\beta^{\prime}}^{\prime}, f_{\beta} ; y\right) \equiv \prod_{j^{\prime}=0}^{e_{\beta^{\prime}}^{\prime}-1}\left(h_{\beta^{\prime}}^{\prime}\left(\zeta^{\prime j^{\prime}} x^{\frac{1}{e^{\prime}}}\right)-h_{\beta}\left(\zeta^{j} x^{\frac{1}{e_{\beta}}}\right)\right),
$$

where Res denotes the resultant and where $\equiv$ means "up to a non zero scalar". Finally, we get

$$
i_{m}\left(\mathcal{C}, \mathcal{C}^{\prime}\right)=\sum_{\beta=1}^{b} \sum_{j=0}^{e_{\beta}-1} \sum_{\beta^{\prime}=1}^{b^{\prime}} \sum_{j^{\prime}=0}^{e_{\beta^{\prime}}^{\prime}-1} \operatorname{val}_{x}\left[h_{\beta^{\prime}}^{\prime}\left(\zeta^{\prime j^{\prime}} x^{\frac{1}{e_{\beta^{\prime}}^{\prime}}}\right)-h_{\beta}\left(\zeta^{j} x^{\frac{1}{e_{\beta}}}\right)\right]
$$




\section{References}

[1] H. Brocard and T. Lemoyne, "Courbes Géométriques Remarquables. Courbes Spéciales Planes et Gauches", Tome I (French) Nouveau tirage Librairie Scientifique et Technique Albert Blanchard, Paris, 1967 viii+451.

[2] J. W. BRUCE, P. J. Giblin and C. G. GiBSON, Source genericity of caustics by reflexion in the plane, Q. J. Math. 33 (1982), 169-190.

[3] J. W. BRUCE, P. J. Giblin and C. G. Gibson, On caustics by reflexion, Topology 21 (1982), 179-199.

[4] J. W. BRUCE and P. J. Giblin, "Curves and Singularities. A Geometrical Introduction to Singularity Theory", second edition, Cambridge University Press, Cambridge, 1992, xviii+321.

[5] F. CATANESE, Caustics of plane curves, their birationality and matrix projections, In: "Algebraic and Complex Geometry", A. Frühbiskrüger (ed.), Proc. Math. Stat., Vol. 71, Springer, Cham, 2014, 109-121.

[6] F. CATANese and C. Trifogl, Focal loci of algebraic varieties. I, Comm. Algebra 28 (2000), 6017-6057.

[7] M. Chasles, Détermination, par le principe des correspondances, de la classe de la développée et de la caustique par réflexion d'une courbe géométrique d'ordre $m$ et de classe n, Nouvelles Annales de Mathématiques (2) 10 (1871), 97-104, extrait C. R. séances A. S. t. LXII.

[8] J. L. Coolidge “A Treatise on Algebraic Plane Curves”, Dover, Phenix edition, 2004.

[9] G. P. DANDElin, Notes sur les caustiques par réflexion, (1822).

[10] J. Dennis LAWRENCE, A catalog of special plane curves, Dover publication Math. Mag. 47 (1974), 103-104.

[11] B. FAnTECHI, "The Evolute of a Plane Algebraic Curve", UTM 408, University of Trento, 1992.

[12] G. FISCHER, "Plane Algebraic Curves", Amer. Math. Soc., 2001.

[13] W. Fulton, "Intersection Theory", 2nd ed., Springer, 1998.

[14] G. H. Halphen, Mémoire sur les points singuliers des courbes algébriques planes, Académie des Sciences XXVI (1889).

[15] R. HARTShorne, "Algebraic Geometry. Graduate Texts in Mathematics", n. 52. SpringerVerlag, New York-Heidelberg, 1977.

[16] A. Josse and F. PÈne, On the degree of caustics by reflection, Comm. Algebra 42 (2014), $2442-2475$.

[17] A. JOSSE and F. PÈNE, Degree and class of caustics by reflection for a generic source, C. R. Acad. Sci. Math. 351 (2013), 295-297.

[18] L. A. J. QUETELET, Énoncés de quelques théorèmes nouveaux sur les caustiques, C. G. Q. 1 (1828), 14, 147-149.

[19] G. SALMon, "Traité de Géométrie Analytique" (courbes planes); destiné à faire suite au traité des sections coniques, 2nd ed., Gauthiers-Villars, Paris, 1903.

[20] G. SAlmon, "A Treatise on Higher Plane Curves: Intended as a Sequel to a Treatise on Conic Sections", Elibron classics, 1934.

[21] C. TRIFOGLI, Focal loci of algebraic hypersurfaces: a general theory, Geom. Dedicata 70 (1998), 1-26.

[22] E. W. von Tschirnhausen, Acta Erud. nov. 1682.

[23] K. UEDA, Pedal curves and surfaces, In: "Mathematical Methods for Curves and Surfaces", Tom Lyche and Larry L. Schumaker (eds.), Papers from the International Conference held in Oslo, June 29-July 4, 2000, Innovations in Applied Mathematics, Vanderbilt University Press, Nashville, 2001, 497-506.

[24] C. T. C. WALL, "Singular Points of Plane Curves", Cambridge University Press, 2004. 
[25] O. ZARISKI, "Le Problème des Modules pour les Branches Planes", course given at the Centre de Mathématiques de l'École Polytechnique, Paris, October-November, 1973. With an appendix by Bernard Teissier, second edition, Hermann, Paris, 1986, x+212.

\author{
Université de Brest \\ UMR CNRS 6205 \\ Laboratoire de Mathématique de Bretagne Atlantique \\ 6 avenue Le Gorgeu \\ 29238 Brest cedex, France \\ alfrederic.josse@univ-brest.fr \\ Université de Brest and Institut Universitaire de France \\ UMR CNRS 6205 \\ Laboratoire de Mathématique de Bretagne Atlantique \\ 6 avenue Le Gorgeu \\ 29238 Brest cedex, France \\ francoise.pene@univ-brest.fr
}

